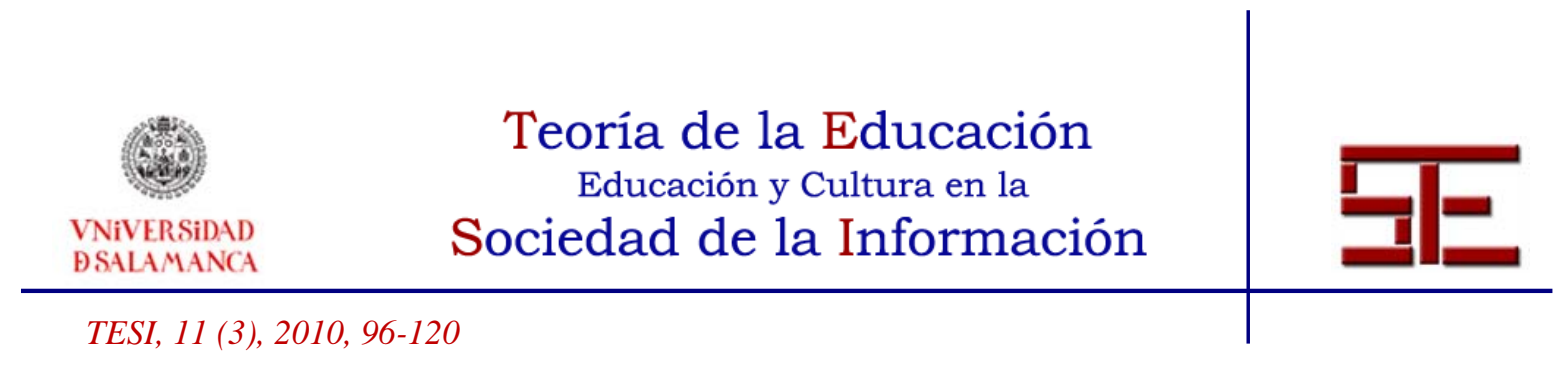

\title{
LOS PROCESOS DE INNOVACIÓN EDUCATIVA EN LA FORMACIÓN UNIVERSITARIA, NUEVOS GENERADORES DE BUENAS PRÁCTICAS EN TECNOLOGÍA EDUCATIVA
}

Resumen:

La influencia de la innovación educativa en la transformación de la universidad española para la Reforma Europea de la Enseñanza Superior es un aspecto crucial. Varios aspectos están haciendo que características del modelo formativo tradicional tengan que ser revisadas a la luz de las demandas sociales y la mejora necesaria de esta formación. Pero este cambio está siendo generado por pequeños grupos de docentes que, imbuidos por la necesidad de transformar su práctica educativa, a partir de pequeños grupos, generan "Comunidades de Práctica" donde reflexionan y toman decisiones sobre buenas prácticas. Las TIC son el eje de dichas mejoras, siendo este trabajo un pequeño esbozo de una de esas experiencias llevadas a cabo por un grupo de personas del Grupo de Investigación reconocido de la Universidad de Valladolid, GSIC-EMIC, asociado a varios de estos grupos de profesores y profesoras, que están trabajando en transformar su práctica diaria desde hace algunos años.

Palabras clave:

Innovación educativa, TIC, comunidades de práctica, buenas prácticas.

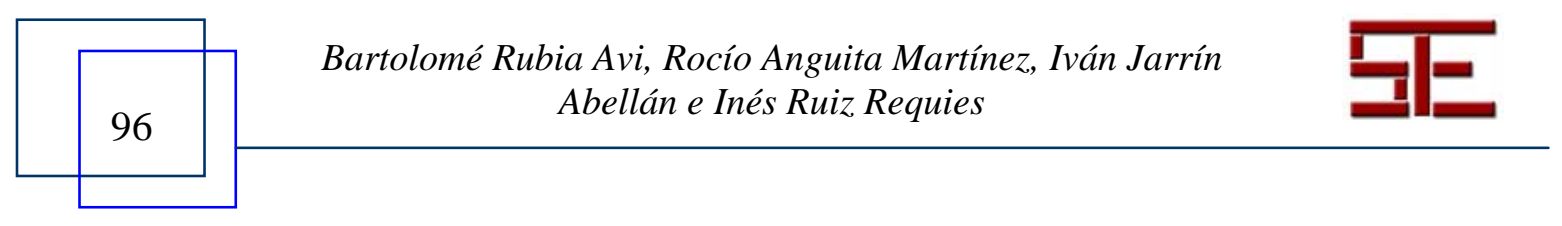




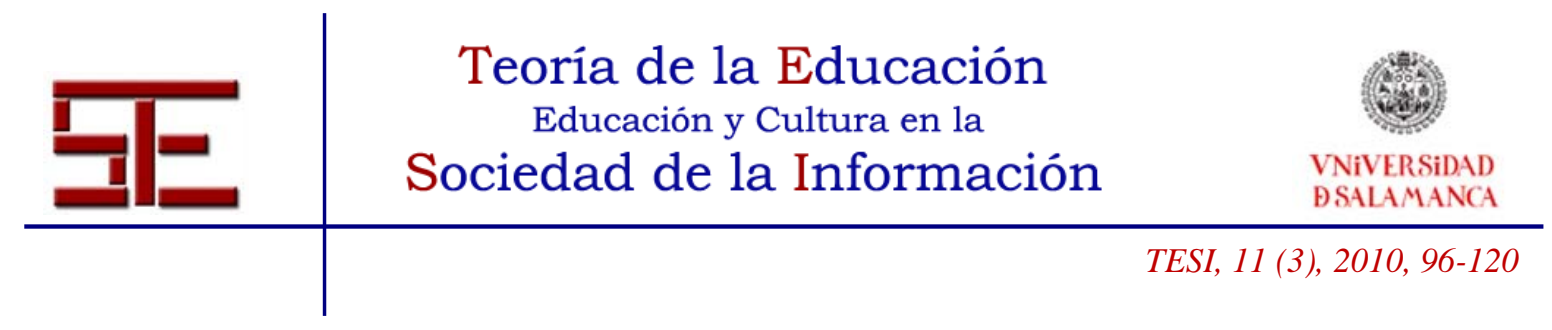

\title{
EDUCATIVE INNOVATION PROCESS IN UNIVERSITY FORMATION, NEW PRODUCTIVE BESTS PRACTICES IN EDUCATIVE TECHNOLOGY
}

\begin{abstract}
:
The influence of educational innovation in the transformation of Spanish Universities to achieved the goals posed by the European Higher Education Area is a crucial aspect for this reform. This process of deep impact within the European countries is promoting the revision of traditional teaching methods. Small groups of teachers and communities of practice are leading this revision by reflecting upon the main issues affecting the higher education system at the same time that they propose horizontal innovations to overcome them. Information and Communication Technologies are becoming of special relevance with regard to the aforementioned innovations. This paper describes a experience conducted within the GSIC-EMIC research team that illustrates the efforts done by a community of teachers at the University of Valladolid (Spain) to analyze and improve their own teaching practices.
\end{abstract}

Key words:

Educative innovation, ICTs, practice communities, best practices.

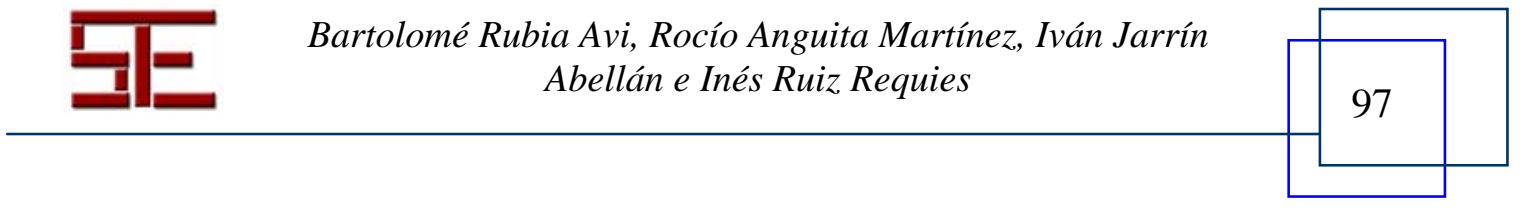




\section{LOS PROCESOS DE INNOVACIÓN EDUCATIVA EN LA FORMACIÓN UNIVERSITARIA, NUEVOS GENERADORES DE BUENAS PRÁCTICAS EN TECNOLOGÍA EDUCATIVA}

Bartolomé Rubia Avi

brubia@doe.uva.es

Universidad de Valladolid.

Rocío Anguita Martínez

rocioan@pdg.uva.es

Universidad de Valladolid.

Iván Jarrín Abellán

ivanjo@doe.uva.es

Universidad de Valladolid.

Inés Ruiz Requies

inesrure@pdg.uva.es

Universidad de Valladolid.

\section{1.- INTRODUCCIÓN}

Este artículo analiza algunos aspectos de la innovación educativa como elemento de cambio en la Universidad española a partir de las medidas tomadas ante la reforma Europea del Espacio de Educación Superior (EEES); atendiendo a la nueva configuración de planes de estudio, así como a los procesos de innovación en las estrategias formativas. Todo ello está transformando una realidad educativa e institucional que permanecía casi intacta desde la Edad Media. Así, estructuras institucionales, dinámicas de organización interna y forma de enseñar (aunque con algunas variaciones propiciadas por las reformas universitarias de los años 70 y los 80, Ley General de Educación y la Ley de Reforma Universitaria) eran un muro impenetrable a los modelos de la modernidad y postmodernidad en educación. Es usual la mención que coloquialmente se suele hacer a la forma de enseñar de un número no despreciable de profesorado que basaba su única

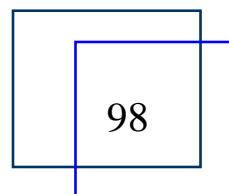

Bartolomé Rubia Avi, Rocío Anguita Martínez, Iván Jarrín Abellán e Inés Ruiz Requies

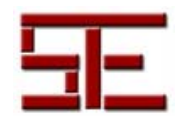




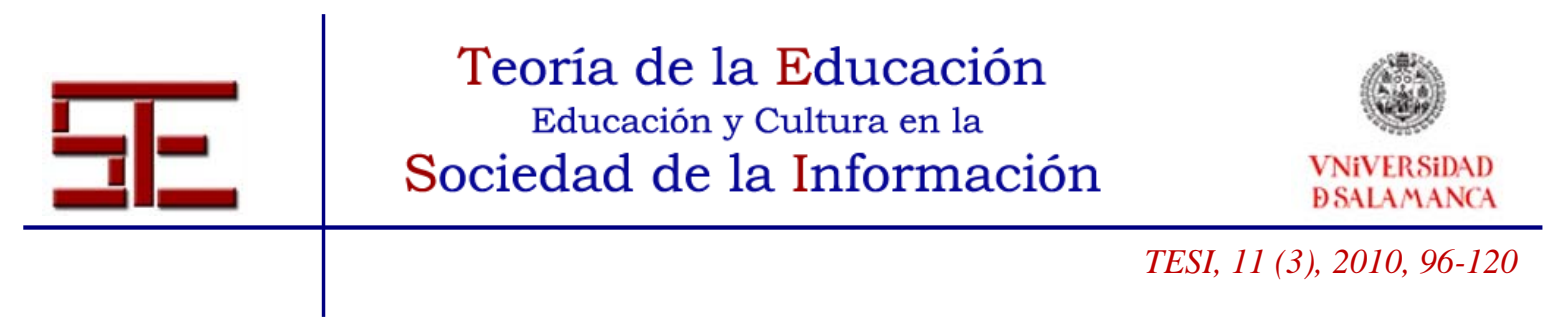

estrategia docente en la lectura de apuntes, que llevaban utilizándose más de una década ${ }^{1}$; o, en el mejor de los casos, el uso solamente de dinámicas expositivas, más o menos bien estructuradas o apoyadas en la capacidad del profesorado para la comunicación, han sido la base de la formación en la Universidad que ahora pretende cambiar. También hay que destacar los momentos en los que parte del profesorado, no todo, realizaba del desempeño de la obligación de atender al alumnado en sus dudas o demandas de formación a través de las tutorías, un espacio tradicionalmente con un cumplimiento laxo.

Por los motivos expuestos y otros similares, en los años 80 y 90, se comienza una primera preocupación por introducir algunos cambios, convirtiéndose los "Trabajos del alumnado” en un pequeño complemento que algunas áreas, sobre todo de Humanidades, incluyen en el desarrollo docente apoyando el estudio de apuntes para un examen.

La tecnología en estas situaciones era un elemento casi inexistente, pero en algunos casos se ha venido usando en algunas materias y estudios, como en las clases de problemas o laboratorios, aunque básicamente no tenían más valor didáctico que el planteamiento expositivo y de resolución de algún tipo de problema sobre la materia. En este tipo de situaciones, era y sigue siendo normal que el profesorado se guarde alguno de esos problemas para el examen donde los docentes sentían y sienten que "cazan a los y las estudiantes incautos", tiernos infantes que no son capaces de solventar la presión de una mente superior, el profesor o profesora, que les está "probando". Todas estas estrategias tenían y tienen por objeto llegar a la evaluación, ese lugar frío en el que el alumnado debe enfrentarse a la emboscada del profesorado, para pasar el filtro de pureza, que en algunos casos les permitía empezar a poseer el marchamo del rito iniciático, donde pasada la formación inicial y superadas las pruebas de oposición, un alumno o alumna podía incluso llegar a ser profesor o profesora. Y así se iniciaba la toma de posesión de un estatus que le permitía, perpetuando el modelo formativo, iniciar los mismos procesos de formación aprendidos por imitación, con sus estudiantes.

Y por último, haremos mención a una parte de los recursos educativos, que con cuentagotas de materiales, empiezan a ser usados teniendo como estrella el retroproyector, un gran salto en el uso de recursos más allá de los puramente expositivos y que lejos estaban de la incorporación de lo que se ha venido entendiendo por Tecnologías de la Información y la Comunicación.

En los últimos 10 años, estamos pasando a una nueva forma de entender la enseñanza en la Universidad. El y la docente ahora no pretende ser un contrario que va a la caza de los incautos que no son capaces de construir su conocimiento, sino que éste pretende

${ }^{1}$ Tal vez dos, es típico y tópico la referencia al color crema de dichos materiales.

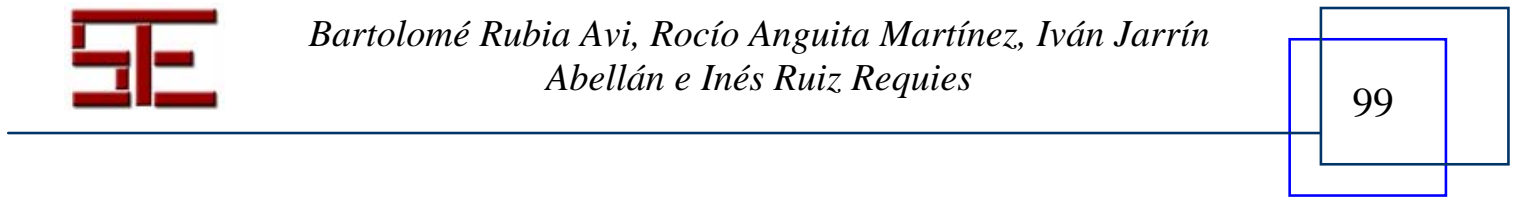




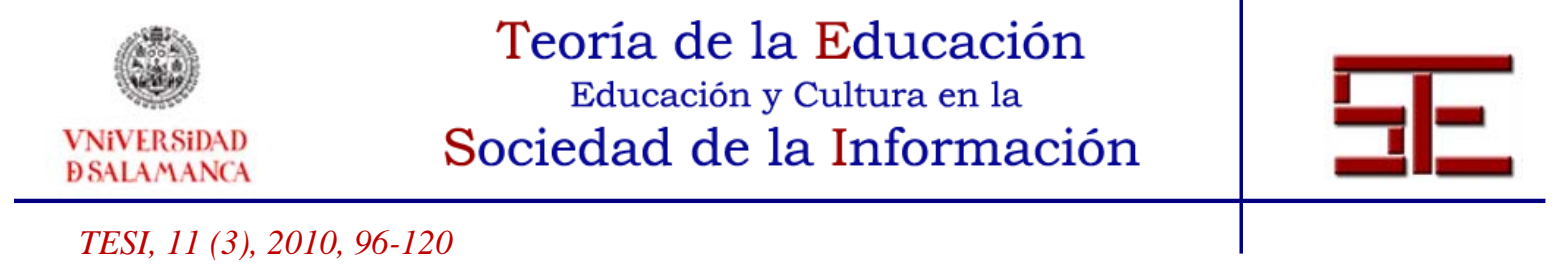

ayudar a sus estudiantes a crear estrategias de aprendizaje, conceptos fundamentales, actitudes sociales y profesionales, además de que lo que aprendan, $\mathrm{i}$ esté conectado con las materias que les rodean en el plan de estudios. Que a su vez comienzan a surgir voces que descubren que dichas asignaturas “tienen mucho que ver con la ellos imparten”. Además de que comienza a cambiar la evaluación sancionadora, por una más formativa.

Y, por supuesto, los recursos tecnológicos, que están suponiendo un gran cambio social puesto que están presentes en todos los ámbitos, se han convertido en un elemento que ejerce presión en la enseñanza superior. Entre otras cosas, en muchos casos son la base de los cambios a través de las exigencias sociales, pero también son un perfecto apellido que suena mejor en la generación de nuevas prácticas.

En este artículo vamos a tratar de presentar una visión sobre la transformación docente que se está produciendo en la universidad española, a partir de las ideas construidas y defendidas por nuestro grupo de investigación en la Universidad de Valladolid, como grupo de innovación e investigación, el grupo GSIC-EMIC ${ }^{2}$. Ideas que nos han servido para construir nuestro trabajo, además de participar en la transformación de la práctica de algunos de nuestros compañeros y compañeras, además de en la formación de muchos de ellos, dentro de nuestra universidad y fuera de ella.

Para ello, mostraremos cómo vemos los procesos de innovación y cambio en la Universidad a partir de la búsqueda de ideas alrededor del concepto innovación, que en este momento tiene un apoyo fundamental en la tecnología y sus procesos de uso.

En un segundo punto, presentaremos las ideas que están alrededor del concepto "buenas prácticas” como eje fundamental para la innovación, incluyendo los elementos que conforman la experiencia de generarlas. También presentaremos nuestra visión sobre los aspectos que forman parte de esas prácticas educativas nuevas en la universidad, así como en lo que entendemos se apoyan.

En tercer lugar, deseamos mostrar nuestra visión sobre cómo debería ser la tecnología que apoyase estos procesos de cambio e innovación, así como algún pequeño debate que se está dando en dicho campo de construcción de recursos para la formación.

Para terminar, vamos a contar lo que ha supuesto un ejemplo de buenas prácticas en formación universitaria, apoyadas en procesos y proyectos de innovación, en el que nuestro grupo ha tenido la suerte de poder participar, analizar y evaluar, poniendo en contacto varias de estas experiencias en nuestra Universidad, apoyándonos en un proyecto de investigación dentro de un programa del MEC, ahora dependiente del MICIN,

\footnotetext{
${ }^{2}$ http://gsic.tel.uva.es/; http://hera.fed.uva.es.
}

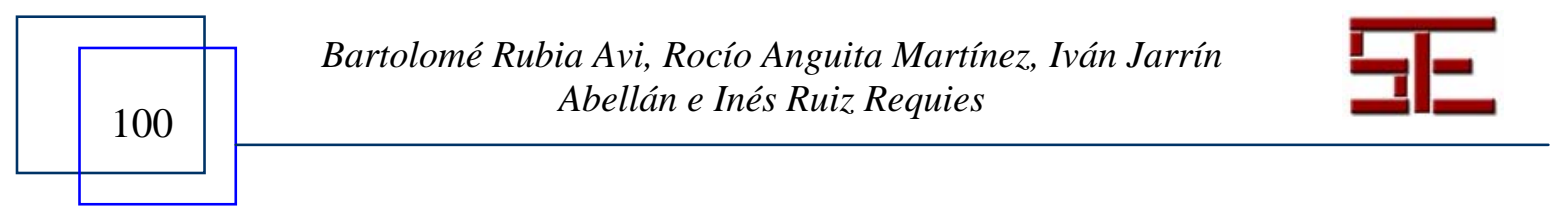




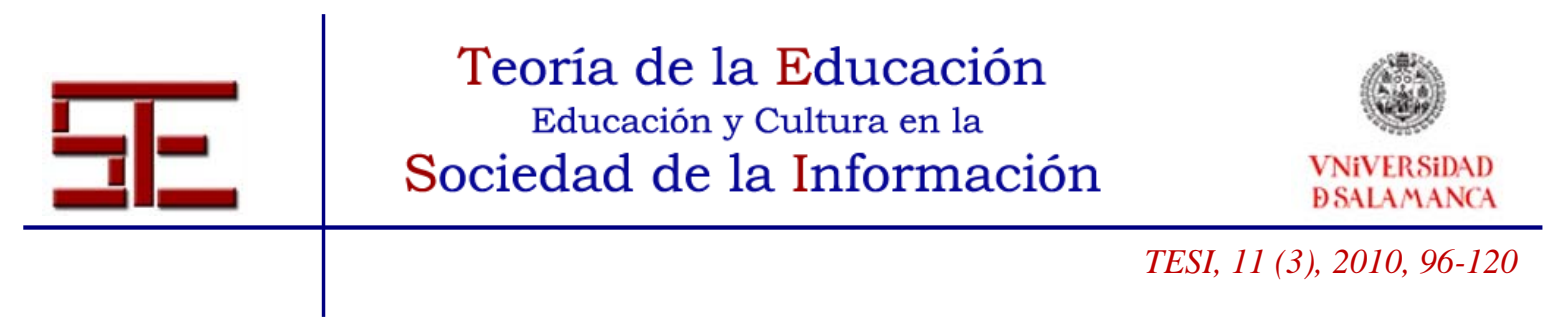

y que tiene como objeto la promoción de esas buenas prácticas. Presentaremos, sobre todo, los aspectos compartidos por esas experiencias de innovación en forma de recomendaciones y decisiones comunes compartidas, todo un ejemplo de transversalidad.

\section{2.- LOS PROCESOS DE INNOVACIÓN Y DE CAMBIO EN LA UNIVERSIDAD}

Si vamos a hablar de innovación, tenemos que empezar por definir este difícil concepto que supone uno de los planteamientos formales de cambio en las reformas educativas desde el principio de los años 90. Así, en la enseñanza reglada no universitaria este concepto adquiere mucho protagonismo, abriendo una dinámica de cambio de discurso y prácticas educativas muy importante. También se convierte en un elemento de confrontación entre los sectores ideológicos tradicional-conservadores, que hasta la fecha en España, desde las épocas anteriores a la implantación del sistema democrático, habían sido el motor de la educación (estructuras organizativas escolares surgidas de las escuelas franquistas y los sectores más activos entre las instituciones de la Iglesia), frente a los colectivos participantes en los Movimientos de Renovación Pedagógica (Sáenz del Castillo, 1999), que habían capitaneado las demandas de cambio de rumbo. Posteriormente, este concepto se convierte en la base de las reformas educativas de la democracia, encontrándose en todas y cada una de las leyes educativas desde entonces.

Etimológicamente, el término se refiere a la introducción de algo nuevo y diferente, entendiéndolo dentro de la educación como un cambio para mejorar. Desde esta perspectiva, el cambio está pensado como el elemento de renovación de una situación educativa que ha de mejorarse.

En el mundo de la educación, atendiendo a la perspectiva de innovación curricular, y citando a José Gimeno en el prólogo del libro L. Stenhouse (1998), los cambios en el currículum han de estar dominados por un proceso de reflexión sobre la práctica. Pequeñas comunidades han de generar un proceso de renovación para la resolución de problemas presentes en dichas prácticas escolares. Es, por tanto, entendible que los cambios que realmente transforman las realidades sociales e institucionales son los que se producen por el consenso de pequeños grupos que, relacionados con otros iguales, van generando una trasformación a través de la reflexión sobre sus propias prácticas.

Los procesos de innovación se apoyan principalmente en la resolución de problemas por parte de todos los miembros de una comunidad. Se caracterizan por la integración de componentes (personales, materiales, estratégicos...) que ayuden a la mejora de la calidad y la eficiencia del proceso educativo. Pero el cambio que plantea una innovación es un proceso planificado, de cara a conseguir algo deseado, y como tal proceso, ha de estar pensado y mensurado para no generar fracaso, puesto que los cambios radicales pueden provocar rupturas entre los agentes que participan del proceso de innovación.

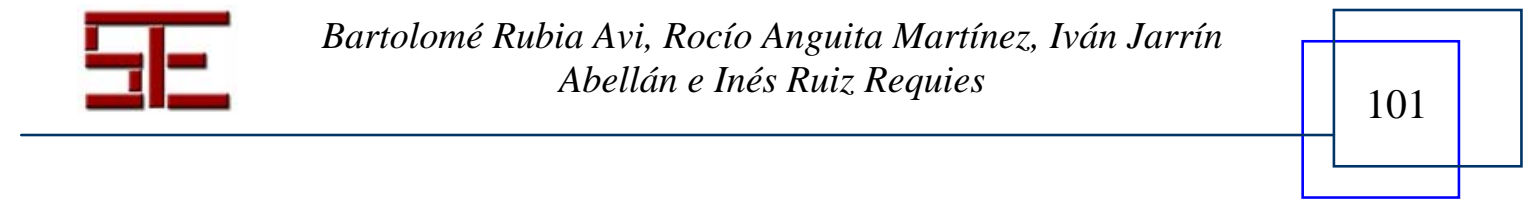




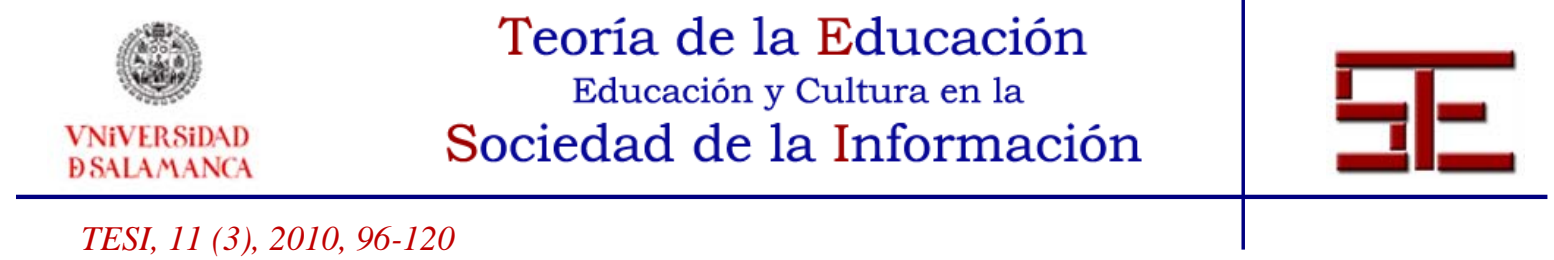

Suelen ser cambios que se producen de abajo arriba, siempre abiertos a la percepción que otros colectivos o personas tienen sobre ellos. Surgen por tanto del profesorado y se apoyan en los conflictos sobre las creencias de los y las docentes para cambiarlas. Presentan diferentes tipos de problemas para su implantación: la centralización institucional, la postura defensiva de los docentes, no tener facilitadores de cambio y la no implicación de madres y padres como ocurre en la formación no universitaria.

Estas características son la base para los cambios y las innovaciones, por tanto, se tienen que producir la mayoría de ellas para que se provoquen los cambios, y la totalidad para que pervivan.

En la Universidad, estos procesos se han desarrollado de manera similar, si nos referimos al cambio que estamos contemplando, porque ese cambio educativo lo están generando pequeños grupos de personas que necesitan mejorar los procesos formativos. Se producen de abajo arriba, puesto que no están apoyados en cambios generales de toda la institución, pero tienen una promesa de cambio obligado con la modificación de los planes de estudio en 2010.

Institucionalmente, son cuatro los compromisos que las Universidades españolas han asumido para cumplir con las demandas propuestas por Bolonia:

1. Creación, desarrollo, transmisión y crítica de la ciencia, la técnica y la cultura.

2. Preparación para el ejercicio de actividades profesionales que exijan la aplicación de conocimientos y métodos científicos o para la creación artística.

3. Apoyo científico y técnico para el desarrollo cultural, social y económico, tanto nacional como de las comunidades autónomas.

4. Extensión de la cultura universitaria.

Es decir, que no se puede quedar sólo en la transmisión de ciencia, sino que deben crearla combinando docencia e investigación, dando sentido práctico y profesionalizador a la formación, así como en contacto con el entorno social, económico y profesional.

La universidad (Connell, 2000) tiene cuatro cometidos específicos, entre los que destaca la innovación, de forma que las universidades se conviertan en focos permanentes de progreso técnico y social. Deben ser capaces de rentabilizar al máximo la autonomía y la disponibilidad de recursos de los que gozan. Desde esta perspectiva, cualquier actuación universitaria debería formar para el desarrollo personal, el desarrollo de conocimientos y competencias concretas y el mejor conocimiento del mundo del empleo para saberse manejar más autónomamente en él. Si entendemos con Salinas (Salinas, 2004) -citando a Toffler (Toffler, 1985)- que los cambios en las organizaciones complejas, como son las universidades, se producen cuando se dan tres condiciones: presión externa importante, personas integrantes insatisfechas con el orden existente y una alter-

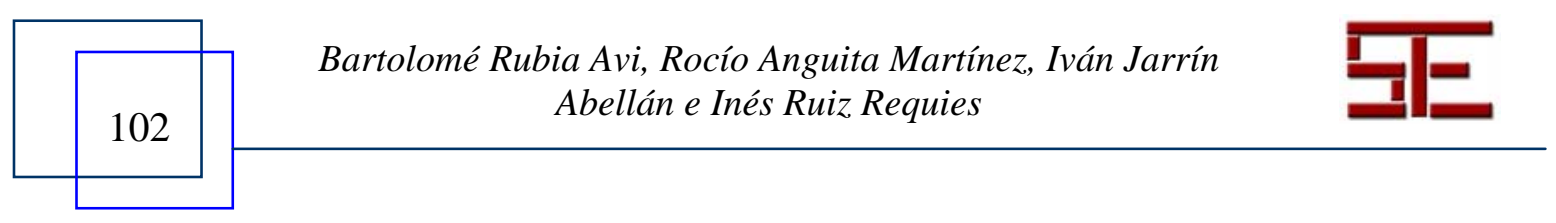




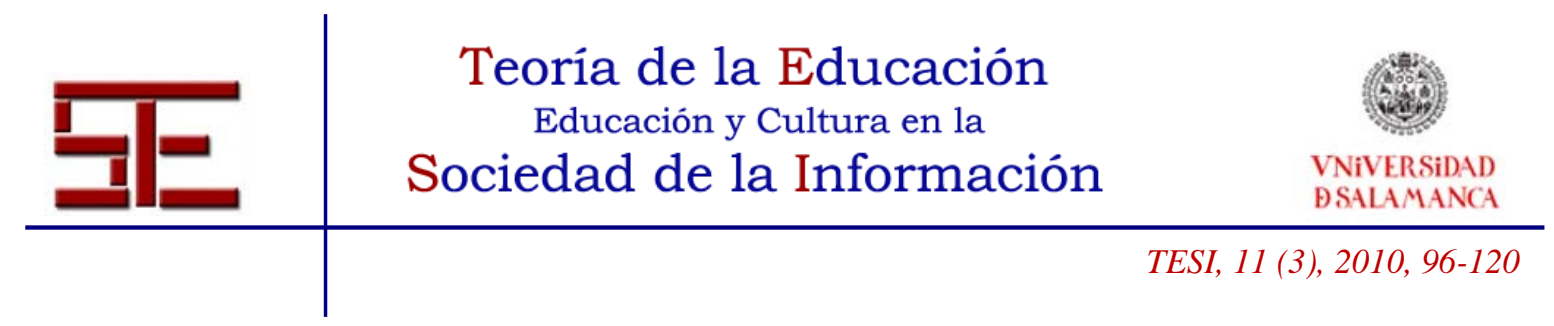

nativa coherente presentada en un plan, modelo o visión. Podemos decir que las obligaciones necesarias de las universidades y la presión externa que reciben desde la "Sociedad del Conocimiento” hacen fundamental que la innovación y el cambio se centren, entre otros aspectos organizativos y de modelos de comunicación, en la integración de Tecnología. No es la reforma de Bolonia la que ha provocado solamente esta presión, porque estas circunstancias se han dado en los últimos 10 años y casi todas estas experiencias de cambio e innovación se han construido alrededor de iniciativas que tenían presente, en el medio de los procesos o como objeto de las innovaciones, las Tecnologías de la Información y la Comunicación (TIC). Lo que conllevan esos cambios y cómo se sitúan las TIC en los procesos formativos es fundamental para el modelo que surja a partir de las nuevas dinámicas, sobre todo, porque las posibilidades de acción que permitan esas tecnologías harán que sean factibles los modelos de innovación educativa que han venido construyéndose y poniendo en práctica, siempre dentro de esas pequeñas comunidades de practicantes y personas involucradas con la formación en la universidad. Es, por tanto, que los planteamientos de innovación se han apoyado más en una concepción no reduccionista, donde se han venido incorporando recursos, personas y procesos de cambio (Salinas, 2004).

Dentro de esta situación, la innovación más exitosa es la que ha facilitado procesos de uso tecnológico amigables, promoviendo procesos educativos que ya venían siendo utilizados. Es decir, si los recursos tecnológicos de la innovación no facilitan la acción educativa como la entienden de manera innovadora los educadores, hacen imposible el cambio y trasladan la acción de enseñar a situaciones no deseadas. Es, por tanto, que si una práctica educativa no es innovadora, no mejora la forma de enseñar y aprender, no tiene valor y produce ineficiencia. Pero si lo es, es lógico que al integrar recursos tecnológicos, ésta práctica no sufra en su desarrollo, siendo el recurso un medio para facilitar la dinámica. En muchos casos, dentro de las dinámicas de cambio reciente en la universidad, los recursos han cumplido esa función de apoyo, en otros han provocando decaimiento en la actitud de cambio y en el valor que le concedemos a los procesos de mejora, haciendo que alumnado y profesorado desistan de ellos.

\section{3.- EXPERIENCIAS DE INNOVACIÓN GENERADORAS DE BUENAS PRÁCTICAS}

Hablar de innovación, es hacerlo de uno de los conceptos que se ha convertido en el talismán de los saberes aplicados en la actualidad, las buenas prácticas en educación. Desde todas las disciplinas podemos observar como el concepto se utiliza para denominar las recomendaciones extraídas de la experiencia y con capacidad de ser exportadas y transmitidas a otras personas que se hallan en un ámbito de trabajo cercano. En esencia no es ni más ni menos que la concepción de la "praxis aristotélica” que tienen todos los ámbitos de conocimiento, pero que en el mundo educativo presentan especial importan-

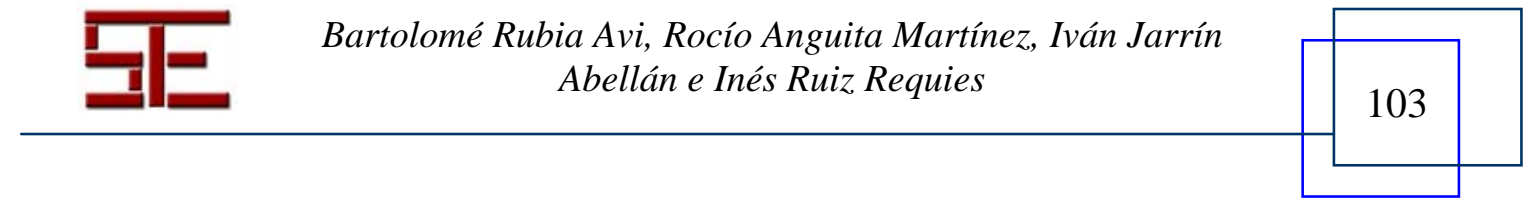




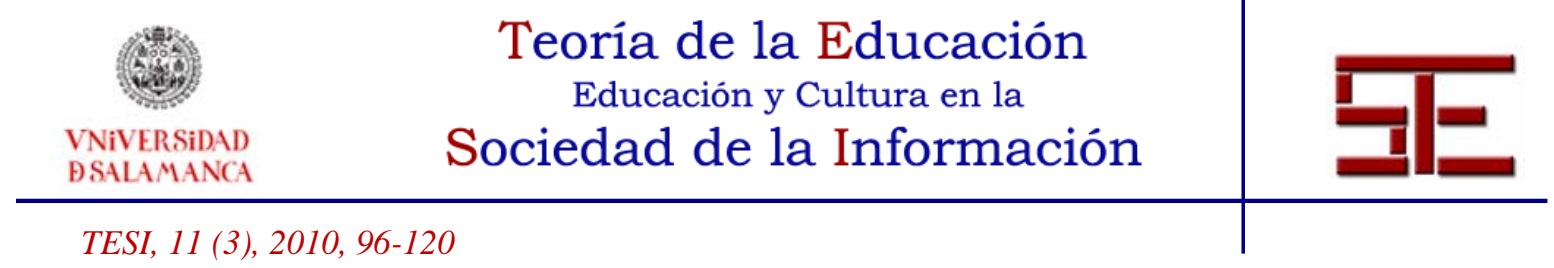

cia (Carr, 1996). En el campo de la educación, el concepto buenas prácticas (Jorrín Abellán et al., 2006) tiene un sentido especialmente importante, porque dicha innovación se produce cuando un educador o grupo de educadores toman decisiones de cambio sobre su práctica educativa para mejorarla, hacerla mejor es el principio básico de una "buena práctica”. Pero este concepto de larga tradición en el mundo educativo y sobre todo en ámbitos de la justicia, la sanidad, etc., ha sido adoptado ahora por muchas áreas de trabajo y conocimiento y nos podemos encontrar publicaciones, portales, buscadores, etc., que lo contienen. Básicamente, lo que incluyen en sus contenidos son recopilaciones de eventos, metodologías, acciones concretas, casos de trabajo, soluciones a problemas concretos que luego se comparten, en definitiva, experiencia acumulada a lo largo del tiempo y la reflexión de muchas personas que trabajan en diferentes ámbitos y situaciones, que han comprendido que la mejor manera de generar saber sobre la forma de llevar a cabo la práctica de una profesión es ayudándose de las experiencias de personas que han vivido situaciones parecidas, que no nos van a dar una solución absoluta para nuestra situación, pero que nos “orientarán” en su resolución.

Esta idea ha ido creciendo y terminado por influenciar también en el mundo de la universidad, sobre todo a través del desarrollo de los programas de cambio en la docencia universitaria por medio de la generación de iniciativas de innovación educativa. Dicho proceso comienza con la puesta en marcha de programas para el desarrollo de Proyectos de Innovación Educativa cuyos precursores fueron las Universidades Politécnicas de Cataluña y de Valencia, iniciadoras de una dinámica que en distintas administraciones o instituciones formativas ha tenido su refrendo. Proyectos de apoyo a la innovación, a la generación de recursos para la formación universitaria o proyectos piloto han sido iniciativas que han supuesto la asunción por un alto porcentaje del profesorado universitario de los programas de cambio en la universidad. Es en el año 2003 cuando el Consejo de Ministros aprueba un plan quinquenal para apoyar el proceso de Bolonia. Se realiza una convocatoria el 27 de enero de 2003 (BOE de 7 de febrero) con la denominación de Programa de Estudios y Análisis destinado a la mejora de la calidad de la enseñanza superior y de la actividad del profesorado universitario. Desde entonces toma cuerpo institucional una dinámica de cambio que en algunos casos ya se venía produciendo, pero que a partir de ese momento recoge las iniciativas de innovación que venían potenciándose en la universidad española.

Este proceso de mejora, cambio e innovación ha tenido un desigual desarrollo en las distintas universidades españolas, creándose espacios en algunas de ellas, que han sido punteras en el desarrollo educativo y tecnológico. Un ejemplo importante en iniciativas de formación e innovación ha sido la Universidad de Alicante ${ }^{3}$, cuya página web se ha convertido en una fuente importante de información. En ella nos podemos encontrar

${ }^{3}$ http://cv1.cpd.ua.es/CatalogaXXI/C10067PPESII1/INDEX.HTML.

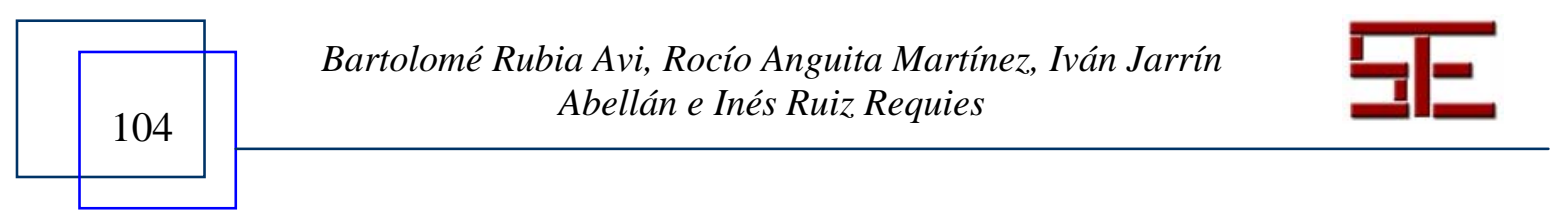




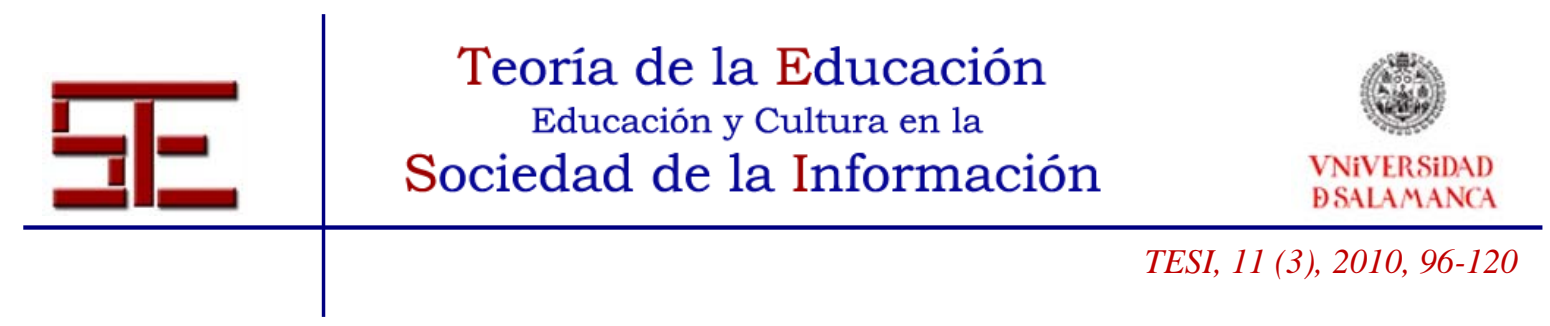

casi de todo en el mundo de la formación del profesorado, la innovación educativa y la integración de tecnología, además de conectar con una serie de servicios distintos sobre contenidos en el ámbito de la tecnología educativa y la educación en el siglo XXI. Podemos decir que en espacios como éste se está creando una fuente de información que conecta a una comunidad de personas e instituciones preocupadas por la formación y la innovación, así como en la integración de tecnología.

Mucha de la experiencia acumulada está dando lugar, también, a una serie de repositorios y fuentes de información sobre las buenas prácticas en el uso de la tecnología como el libro virtual "Libro de Buenas Prácticas de e-learning”, , promovido por la Universidad a Distancia de Madrid y que recoge planteamientos generales sobre el uso de la tecnología en la formación, así como un buen repositorio de buenas prácticas. Dentro de esta dinámica de formación y cambio, nosotros hemos vivido la experiencia de la ebullición de este tipo de procesos y donde se ha puesto en marcha multitud de proyectos piloto, así como programas de formación de profesorado que ha facilitado la innovación y el cambio en la Universidad de Valladolid, siendo ésta evaluada de manera experimental en 2005 por la ANECA. Si analizamos estas transformaciones, tanto en nuestro entorno cercano, como en los espacios ya mencionados donde se recogen esta "euforia de transformación” o “necesidad de ser buen docente” como plantea De la Calle (2004), el cambio se ha centrado en varios aspectos fundamentales que sintetizados podríamos identificar como:

a.- Cambios de materiales formativos. Quizás la primera de las transformaciones que acomete un docente, cuando se preocupa por lo que está haciendo en su clase y comienza a necesitar cambiar algo en su proceso de formación. En ese momento, comienza a buscar nuevos recursos para el desarrollo de la formación que está llevando a cabo.

b.- Cambios de dinámicas de formación y rol del profesor. Cuando continúa en la dinámica de transformación de su actividad, el profesorado comienza a cuestionarse el rol que representa y la forma que tiene de generar los procesos comunicativos que pone en marcha en su clase y necesita modificar la actitud que tiene ante sus alumnos, así como la que estos últimos tienen ante él. La preocupación en este momento se centra en superar el rol pasivo del alumnado y hacer que éstos tomen una responsabilidad mayor en los procesos de aprendizaje. Para el profesorado esto supone que ha de modificar la forma de comportarse en clase, la asunción de responsabilidades y la función, que en este caso pasa a ser más de facilitador del aprendizaje, frente a la clásica de postura de "contenedor del conocimiento" o dador del mismo.

\footnotetext{
${ }^{4}$ http://www.buenaspracticas-elearning.com/.
}

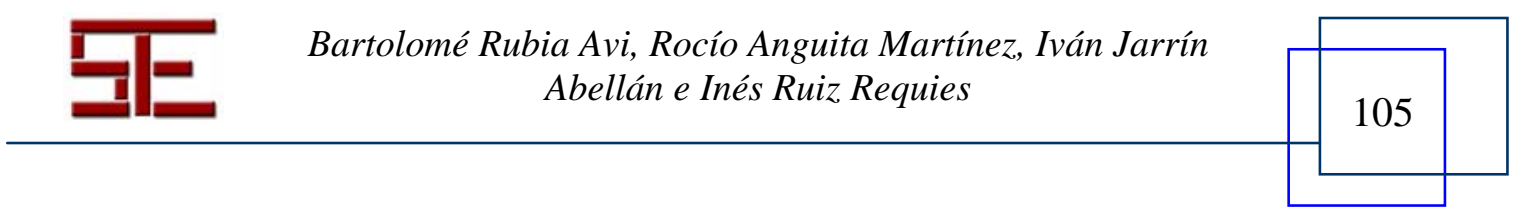




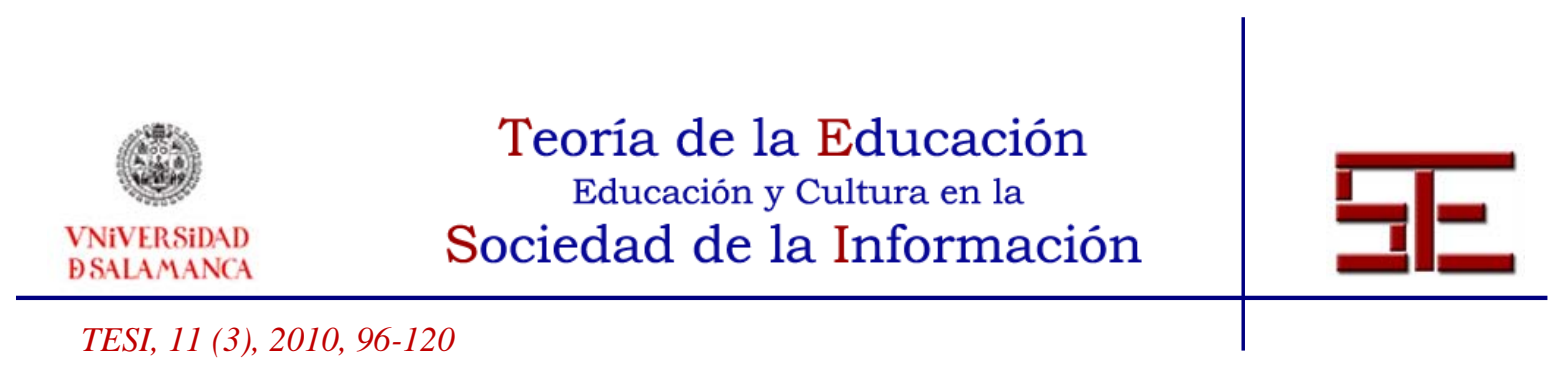

c.- Cambios en la relación con sus iguales. Es uno de los aspectos fundamentales, puesto que una de las transformaciones más evidentes tiene que ver con la modificación de la visión de la cultura universitaria. El profesorado tiende a buscar la pervivencia de sus innovaciones en la complicidad de sus iguales, por tanto, es usual que se produzcan los cambios propiciados por grupos de profesores unidos por la necesidad de modificar y mejorar su docencia.

d.- Cambios de apoyos tecnológicos. Y por último en estos procesos de transformación, aunque a veces suele ser la preocupación sentida que lleva al docente a acometer los anteriores, la búsqueda de apoyos tecnológicos que faciliten los nuevos procesos formativos, que habitualmente tienen necesidades de abrir la posibilidad de trabajo en las asignaturas durante las 24 horas del día, facilitar la comunicación on-line y off-line con el alumnado modificando el concepto tutoría, posibilitar el uso de recursos formativos de diversos tipos de manera permanente, no sólo durante las clases formales de la universidad, posibilitar espacios de trabajo permanente donde el alumnado pueda realizar, de manera individual o en grupo, tareas que puedan ser supervisadas por el profesor de igual manera, todos en un entorno on-line.

Éste fue el caldo de cultivo donde nuestro grupo de investigación ${ }^{5}$ ha centrado sus esfuerzos en los últimos años, realizando diferentes propuestas y experiencias de innovación (González et al., 2007; González, et al., 2008; Jorrín Abellán et al., 2006; Martín et al., 2007; Martínez-Mones et al., 2003; Rubia-Avi et al., 2004; etc.) dentro de la responsabilidad formativa que tenían algunos profesores y profesoras pertenecientes al grupo, así como ayudando, asesorando e investigando otras experiencias en el ámbito de la formación universitaria que se ponen en marcha durante los últimos años.

Desde nuestra experiencia, llevamos años trabajando con grupos de personas y colectivos de enseñantes en distintas materias que han desarrollado alguna de estas buenas prácticas, así como cambiado la forma de llevar a cabo la formación. Todo ello ha dado lugar a la plasmación de nuestros esfuerzos dentro de un proyecto de investigación centrado en el programa de Estudios y Análisis, anteriormente mencionado, y que será un ejemplo que analizaremos más adelante en el punto 4.

\section{4.- TECNOLOGÍA COMO EJE VERTEBRADOR DE LAS INNOVACIONES ACTUALES}

Antes de analizar nuestra práctica o la referencia sobre algunas de esas "buenas prácticas” en las que hemos participado y que se han recogido durante años en las publicaciones ya citadas, nos gustaría hacer mención a un aspecto transversal que las han unido en

\footnotetext{
${ }^{5}$ GSIC-EMIC (http://ulises.tel.uva.es http://hera.fed.uva.es).
}

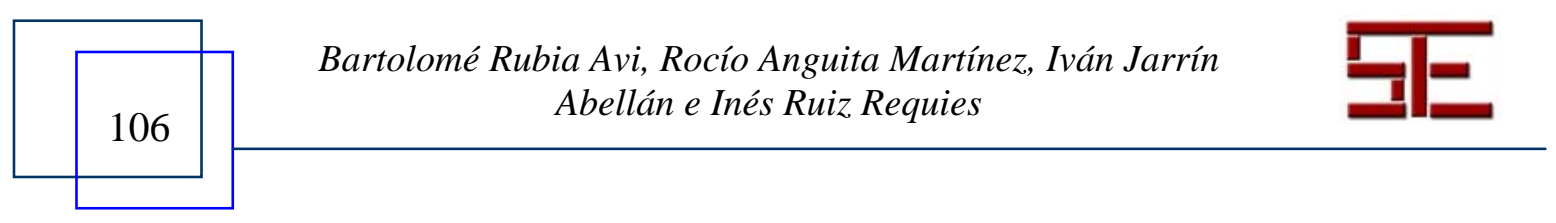




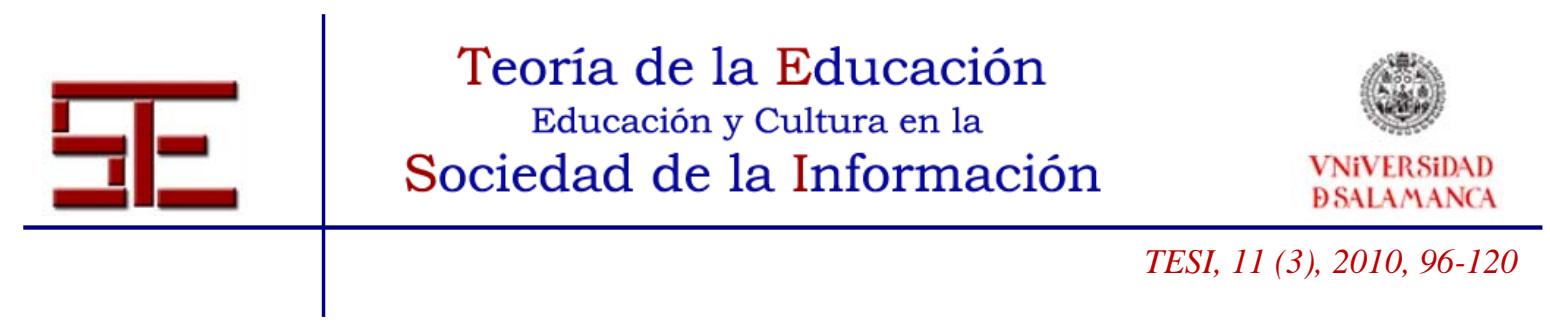

gran medida. Casi todas han tenido un aspecto de apoyo fundamental en la integración de la tecnología en el desarrollo del proceso formativo. Pero esta experiencia en la definición de espacios tecnológicos para apoyar el proceso de innovación nos ha hecho preguntarnos muchas cuestiones sobre qué tipo de tecnología hemos de usar y cómo habría que configurarla (Jorrín Abellán et al., 2008). A veces, el planteamiento tecnológico nos ha llevado a adaptar tecnología existente (Rubia-Avi et al, 2004; Jorrín Abellán et al., 2006) y, otras veces, hemos construido nuevos recursos desde las necesidades expresadas por las prácticas eductivas (Gómez et al., 2002; Hernández-Leo et al., 2006; Bote et al., 2008; Vega et al., 2008).

De entre todas las experiencias adquiridas podemos sintetizar algunas ideas sobre cómo debe ser el apoyo que tiene que hacer la tecnología en estos procesos formativos:

a.- No toda la tecnología sirve para cualquier espacio educativo, ni para la implementación de la docencia en todas las áreas de conocimiento. Es fácilmente entendible que los requerimientos educativos están condicionados por el área de conocimiento a la que nos refiramos. Hay acciones educativas que requieren un tipo de espacio o tecnología distinta a otra. No es lo mismo enseñar física, con la necesaria implementación de un laboratorio virtual, que no tiene los mismos requerimientos que la filosofía, con la definición de espacios tecnológicos distintos a la anterior.

b.- No podemos cambiar nuestro modelo docente por la integración de las TIC. A veces, la integración de recursos tecnológicos hace que los modelos educativos se transformen, por tanto, no podemos usar tecnología sin mantener nuestros principios pedagógicos. No podemos pasar de un proceso de aprendizaje colaborativo a uno individualizado, simplemente porque se transforme nuestra estructura de clase por la tecnología y, por supuesto, al revés tampoco.

c.- Cada espacio tiene su tecnología. Es por tanto, que no todos los niveles educativos ni en las situaciones contextuales de la educación se deben usar el mismo tipo de tecnología, ni en número ni en forma o uso. Las necesidades de las personas determinan las tecnologías, pero las necesidades educativas son las que tienen que definir el tipo de tecnología que hay que crear.

d.-El diseño y desarrollo de la misma se debería de hacer en colaboración con tecnólogos, por parte del profesorado. Esta máxima es fundamental, a veces, la educación ha usado recursos tecnológicos nacidos para usos no educativos, esto ha supuesto ventajas, pero básicamente inconvenientes por la transformación del sentido educativo que han tenido en sus aplicaciones.

Todas estas recomendaciones y aspectos generales extraídos de nuestra experiencia nos han permitido entrar en un debate sobre el tipo de tecnología, cómo usarla y quién debe o no decidir cómo ha de configurarse. Y aunque hayamos mencionado todos estos ele-

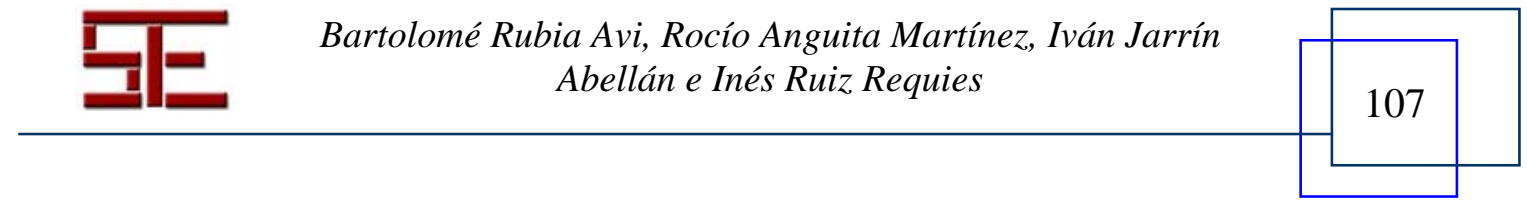




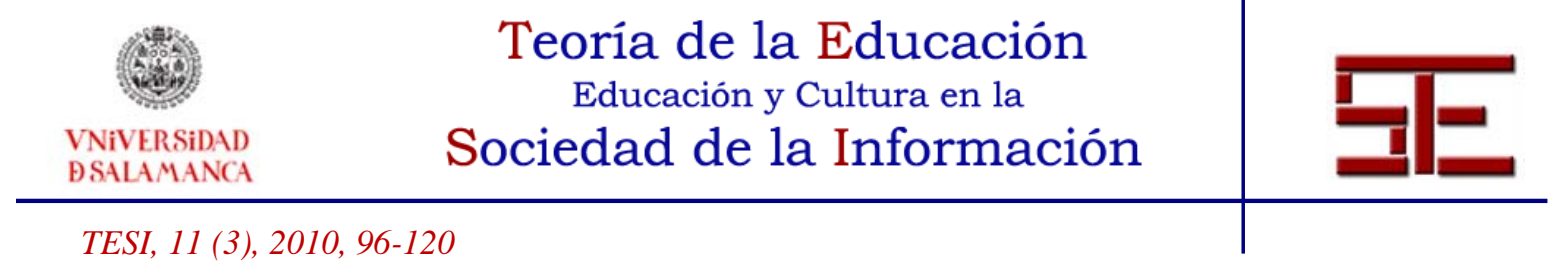

mentos relacionados con los aspectos que a nosotros nos han aportado las buenas prácticas, sí que podemos argumentar que sí es posible definir elementos reutilizables tanto en el ámbito de la educación como de la tecnología. Así, podemos identificar una serie de estándares educativos y tecnológicos que están consiguiendo la definición de modelos educativos compartidos por una amplia comunidad internacional, así como estándares tecnológicos, en los que se encuentran implicados multitud de personas que se dedican a su desarrollo.

\section{1.- Estándares educativos}

Si por estándar podemos entender lo que sirve como tipo, modelo, norma, patrón o referencia, aplicable a múltiples contextos, en los educativos se está generando uno relacionado con la utilización de metodologías comunes, que se pueden adaptar a circunstancias distintas. Un ejemplo fundamental, y que para nosotros es la fuente de todo nuestro trabajo, tiene que ver con el Computer Supported Collaborative Learning (CSCL) (Koschman, 1996). Dentro de esa concepción educativa del aprendizaje, se han establecido, a raíz de las buenas prácticas en el uso de tecnología para la formación, una serie de técnicas de trabajo colaborativo que se han convertido en el objeto de implementación de muchos grupos y personas que trabajan en este ámbito. Lugar reconocido y reconocible entre esta comunidad es la web del National Institute for Science Education (USA) ${ }^{6}$ que resume de forma excelente cada una de las técnicas que se han convertido en un referente dentro de este campo de la investigación y la formación.

\section{2.- Estándares tecnológicos}

De igual manera, en el ámbito tecnológico, también hay abierto un debate fundamental que tiene por objeto la definición de estándares que sean reutilizables en cuanto a los planteamientos educativos y tecnológicos. Así, la definición de "objetos de aprendizaje” está implicando a organizaciones como la IEEE (Learning Technologies Standardization Committee, IEEE LTSC), cuyo principal objetivo se centra en el desarrollo de estándares técnicos, prácticas recomendadas y guías para componentes software, herramientas, tecnologías y métodos de diseño que faciliten el desarrollo, implantación, mantenimiento e interoperatividad de sistemas software de enseñanza-aprendizaje. Otra opción tiene que ver con el consorcio global para el aprendizaje IMS (Innovation Adoption Learning), una comunidad de fabricantes de hardware y software, instituciones educativas, editoriales, agencias gubernamentales, integradores de sistemas, proveedores de contenidos multimedia y otros consorcios de menor tamaño, es actualmente la iniciativa más activa en el desarrollo de especificaciones y estándares en este dominio. ADL (Advanced Distributed Learning) trata la educación basada en webs, coordinando

\footnotetext{
${ }^{6}$ http://www.wcer.wisc.edu/archive/CL1/CL/doingcl/DCL1.asp.
}

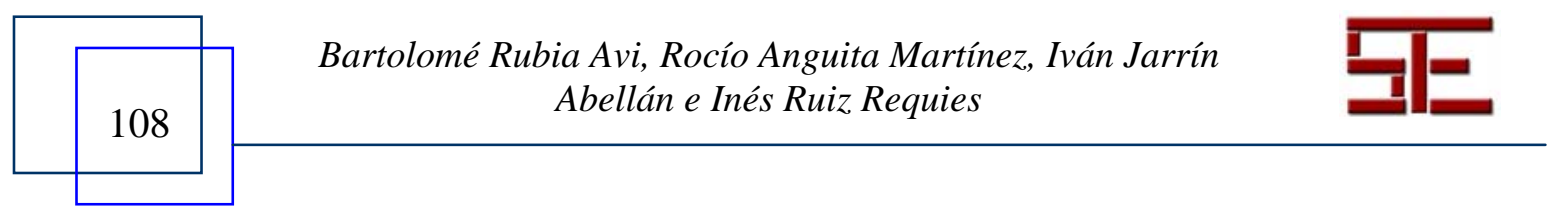




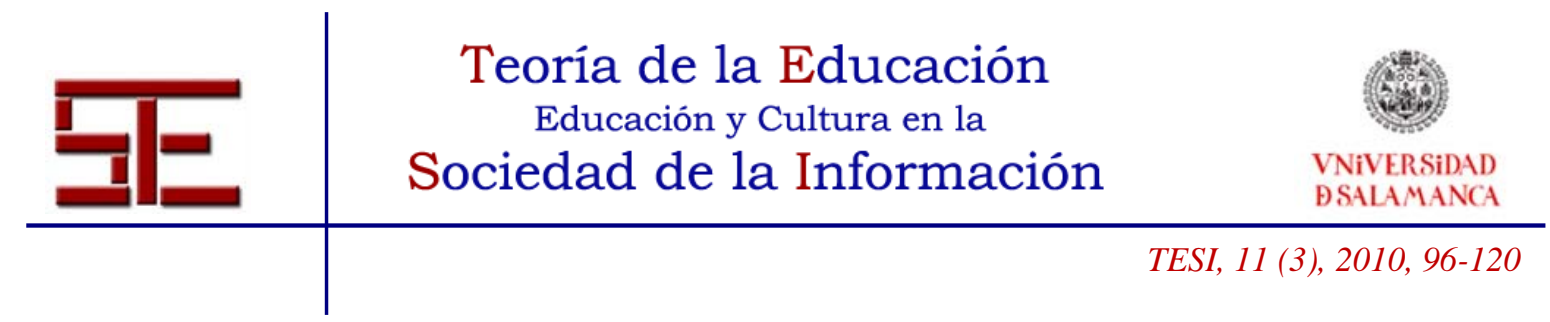

sus actividades con las de otras organizaciones como IEEE, IMS y AICC. Su trabajo más importante es el modelo de referencia SCORM, propuesta que engloba un modelo de referencia para objetos de aprendizaje, un entorno de ejecución y un modelo de agregación de contenidos orientado al uso compartido de los mismos. Y en Europa organizaciones como Prometeus (Promoting Multimedia access to Education and Training in the European Society), GESTALT(Getting Educational Systems Talking Across Leading edge Technologies), etc., están realizando labores similares a las mencionadas anteriormente (Sicilia et al., 2006).

Ambas aproximaciones están relacionadas con un cúmulo de buenas prácticas adquiridas por comunidades de enseñantes e investigadores en computer science que comparten y trabajan desde perspectivas comunes para el desarrollo de espacios compartidos para apoyos a la enseñanza.

\section{5.- UN EJEMPLO DE INNOVACIÓN EDUCATIVA CON APOYO TECNOLÓ- GICO, ENMARCADO EN EL ÁMBITO DE LAS BUENAS PRÁCTICAS}

Como miembros de una comunidad de enseñantes, pertenecientes a las áreas de educación y telecomunicación, hemos ido construyendo un grupo que ha generado y compartido múltiples experiencias que han generado buenas prácticas. Desde hace más de 10 años, nuestro grupo ha colaborado con una parte importante de personas interesadas en la innovación y el cambio educativo, con apoyo tecnológico. Así pues, en este tipo de relaciones, surgió un proyecto dentro del Programa de Estudios y Análisis del Ministerio de Educación y Ciencia en su convocatoria del año 2006, a la luz de las colaboraciones y relaciones de nuestro grupo con otros implicados en la innovación educativa en la Universidad.

\section{1.- El contexto de innovación}

Nuestro trabajo se centró en el análisis de uno de esos entornos de cambio en la formación universitaria, que surge de las propias bases del profesorado y del alumnado que no está conforme con una estructura tradicional. Concretamente, se enmarca en la Universidad de Valladolid, apoyado en la dinámica de formación e innovación puesta en marcha con la perspectiva de mejorar para adaptarse al EEES. Y dentro de este espacio de trabajo educativo, de formación e innovación, en un grupo de personas de distintas titulaciones interesadas por la mejora de su práctica docente enfocada hacia la formación colaborativa y grupal, así como cercana a la realidad.

También pretendió extraer conclusiones sobre aspectos de la formación apoyados en procesos de innovación educativa, pero igualmente desde una posición inductiva de las buenas prácticas. Esto supone que las conclusiones sobre la innovación en las experien-

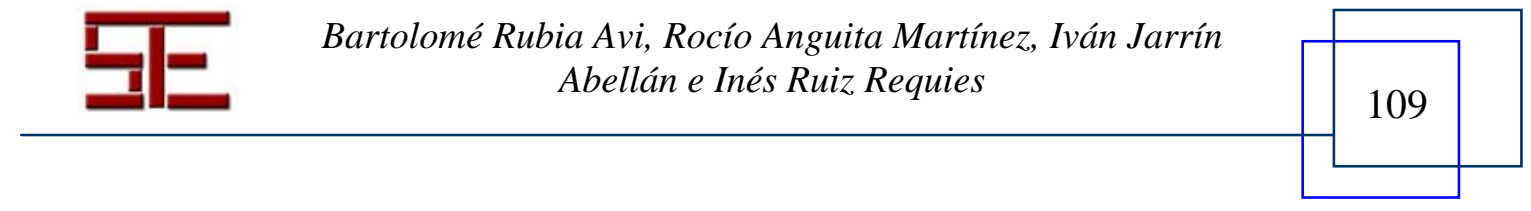




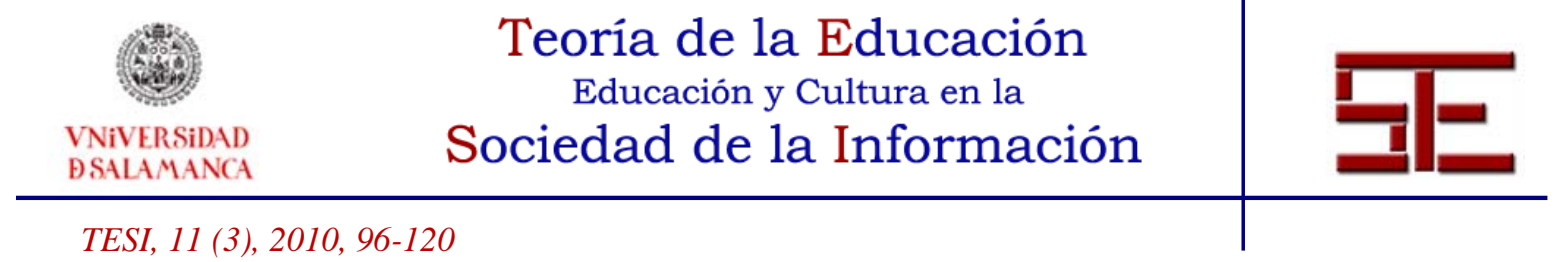

cias presentadas en este ejemplo han sido las generadoras de ideas concretas sobre cómo debería realizarse de manera eficiente un proceso de este tipo. Realizadas desde distintas y distantes formas de entender la formación y la innovación, así como el uso de tecnología en ellas, pero todas ellas comparten un planteamiento de cambio y también la perspectiva de la integración de las Tecnologías de la Información y la Comunicación (TIC) para mejorar y facilitar el proceso formativo. Este análisis compartido a partir de las respectivas experiencias que concurrieron desde sus buenas prácticas se desarrollaron en:

- $\quad$ 4. ${ }^{\circ}$ de Ingeniería Química en la Facultad de Ciencias de Valladolid

Un proyecto de innovación educativa que incorpora a toda la titulación de la carrera y a la mayoría del profesorado, pero que inicialmente comenzó generando una propuesta de cambio en el curso $4 .^{\circ}$ de la titulación.

- 1 1. $^{\circ}$ Maestro especialista en Educación Primaria en la E.U. de Educación de Soria Igualmente, proyecto de innovación educativa $\mathrm{y}$, posteriormente, proyecto piloto de innovación para el espacio europeo en la Universidad de Valladolid, que esencialmente se centra en el desarrollo de un plan de integración de la titulación en el desarrollo de competencias educativas, así como la implementación de un proceso de reconversión de la titulación, involucrando también a todo el profesorado.

- $\quad 1,{ }^{\circ}$ de Trabajo Social en la Facultad de Educación y T. S. de Valladolid

Como la anterior, un proyecto piloto de integración de la titulación en el EEES, involucrando a todo el profesorado y dentro de los proyectos piloto de la Universidad de Valladolid. Caracterizado por la creación de un nuevo entorno docente, cambiando la forma de llevar a cabo las actividades formativas y centrándolas en el trabajo en grupo.

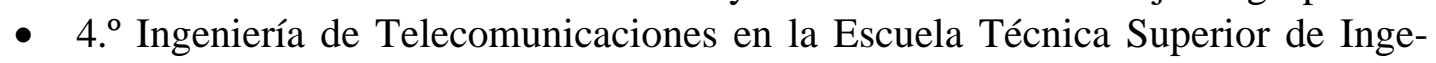
niería en Telecomunicación de Valladolid

Este caso fue una de las primeras experiencias que en la UVA se plantearon la reforma de la docencia para la formación de ingenieros en Telecomunicación, convirtiéndose en proyecto de innovación educativa que ha servido para explorar y potenciar el Project Based Learning (PBL) y los procesos de aprendizaje colaborativo. En este caso sólo está implicado el grupo de profesores que imparte la asignatura Arquitectura de Ordenadores, pero, posteriormente, el esquema de trabajo se ha extendido por varias asignaturas de la titulación.

- Asignaturas de Física I, Física II y Laboratorio de Física de Ingeniería Industrial dentro de la Escuela Técnica Superior de Ingeniería Industrial de Valladolid.

Este caso ha sido proyecto de innovación educativa, promovido por la Junta de Castilla y León como los anteriores, centrándose en la innovación de una asignatura de primer curso de la carrera, que intenta promover dinámicas de trabajo en grupo, así como el apoyo en entornos tecnológicos. En este caso está implicado todo el profesorado de la asignatura, un total de 5 .

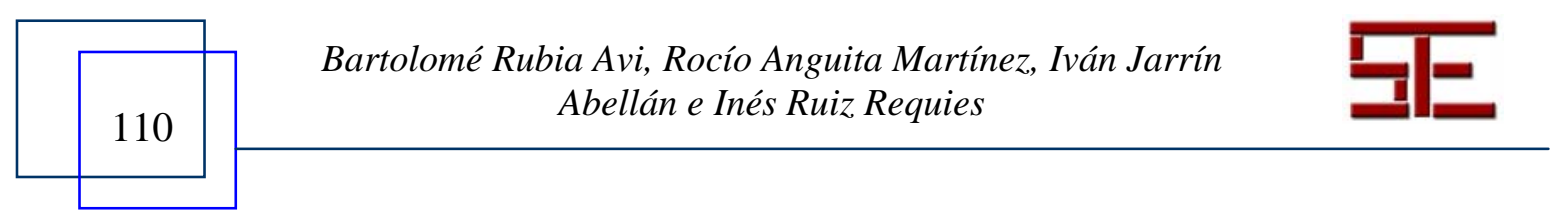




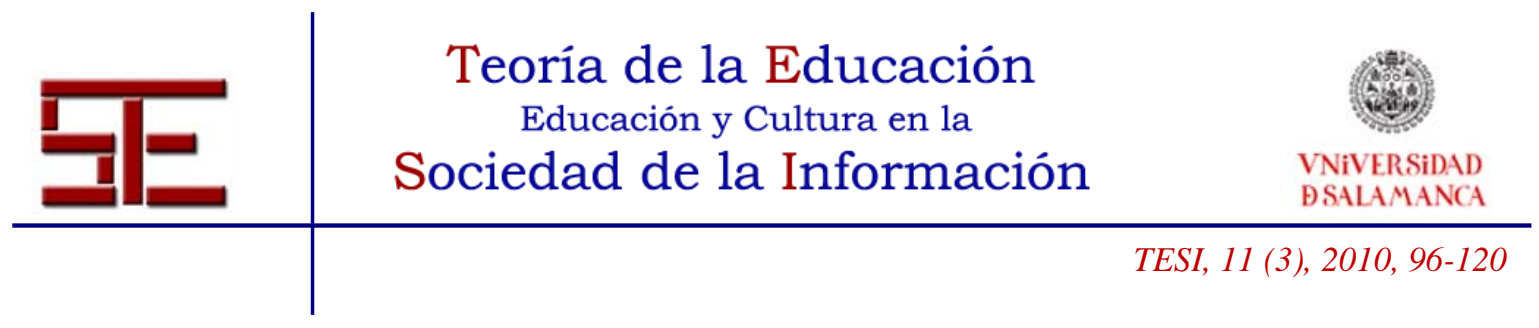

- Nuevas Tecnologías Aplicadas a la Educación en la Facultad de Educación y Trabajo Social de Valladolid

Y, por último, esta asignatura que se encuentra en el plan de estudios de todas las titulaciones de Magisterio y Educación Social, que también ha sido igualmente proyecto de innovación de la Junta de Castilla y León, nos plantea un caso de asignatura que se integra dentro de los procesos de aprendizaje grupal, PBL y apoyos tecnológicos, para el desarrollo de un proceso colaborativo de aprendizaje. En ella se han integrado de manera transversal todo el profesorado implicado (cuatro profesores) que la imparten en todas las titulaciones de magisterio y en la titulación de Educación Social.

\section{2.- El proceso de intercambio y generación de ideas para la innovación}

En este apartado, vamos a reflejar el ejercicio de reflexión para analizar las prácticas compartidas en las diferentes experiencias de formación e innovación mencionadas en el subapartado anterior. Parte del concepto transversalidad, que es un ejercicio de relación y acercamiento de problemas y aciertos, virtudes y desventajas que posee cualquier acción práctica. Cuando una comunidad de enseñantes se reúne para contarse lo que hacen, plantean, piensan, resuelven y comparten muchas cosas de su realidad y de los demás, cada uno de ellos puede extraer ideas propias para luego integrar en esa práctica, no podrá trasladar de manera directa las mismas soluciones a problemas parecidos pero diferentes, pero sí que podrá aprender de la experiencia de otros, aplicando soluciones nuevas ideadas por él, a la luz de las reflexiones de sus compañeros y compañeras de viaje, porque las realidades sí son compartidas. Este intercambio surgido del trabajo en este proyecto lo dividimos en los siguientes apartados y aspectos que determinan los procesos de innovación:

\section{A. APORTACIONES A LOS PROCESOS DE INNOVACIÓN}

El primer punto de análisis tiene que ver con las aportaciones que hace este tipo de procesos de innovación a la mejora de la práctica formativa. Aspectos que tienen que ver con el nivel de satisfacción que producen, aunque generen una alta carga de trabajo. Dentro de los grupos de personas implicadas en la innovación se ha iniciado la construcción de "comunidades de práctica”, conjuntos de personas que piensan, deciden, actúan juntas y van creando un lenguaje común. De hecho, las relaciones personales son el principal apoyo para el buen funcionamiento de estas dinámicas, además del caballo de batalla sobre el que se aposentan los cambios. También se puede decir que el nivel de satisfacción del profesorado con el rendimiento del alumnado es otro de los aspectos de mejora.

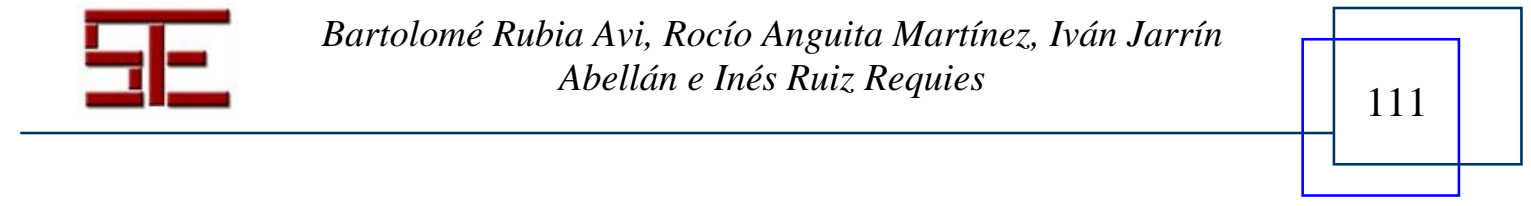




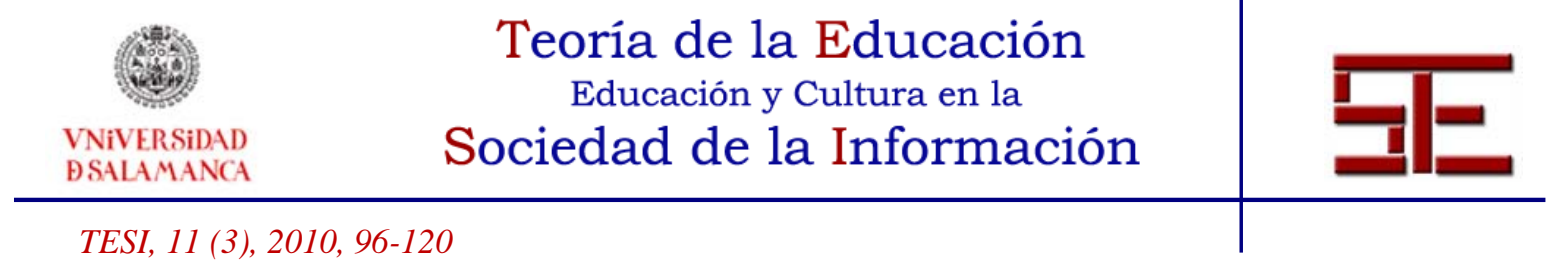

Por otra parte, observamos que este nivel de actividad tiene un problema de sostenibilidad. Es difícil mantener en el tiempo una dinámica así, sin contemplar más incentivo que la mera satisfacción personal. La preocupación por los procesos y los resultados es un aspecto que empieza a implantarse, frente a la tradición de buscar sólo los resultados. Por tanto, la innovación es considerada por las personas que la llevan a cabo como la vía para el cambio, suponiendo un aspecto fundamental de autorreflexión sobre la propia actividad de los docentes. Y uno de los aspectos más reconocidos de entre las distintas experiencias tiene que ver con la mejora fundamental de la comunicación entre profesor-profesor, profesor-alumno y alumno-alumno. Para esto es importante buscar la resolución de problemas entre los intereses de los profesores y los alumnos, de cara al avance de la innovación. Pero vayamos de manera pormenorizada a los aspectos que surgieron de esta experiencia de análisis compartido de innovaciones y buenas prácticas:

\section{Dinámicas de trabajo en grupo e individual}

Este aspecto ha sido fundamental en todas las metodologías innovadoras puestas en marcha en las distintas experiencias, haciendo importante su traslación a la conciencia del alumnado, cuestión fundamental para su éxito. Es fundamental cuando se incluye una nueva metodología la necesaria información al alumnado de sus hitos y pasos, de su filosofía e intereses, así conseguimos que se interiorice y facilite los procesos de interacción. Para esto, es indispensable que exista una formación previa para llevar a cabo esta metodología, por parte del profesor, con todo el alumnado. Es necesario, también, que el profesor esté formado en dinámicas de grupo, siendo muy consciente de lo que supone el proceso de interacción entre los alumnos. Es igualmente fundamental conocer cuál es la mejor forma de trabajo en grupo y de manera más eficaz, elemento significativo en la experiencia piloto de Trabajo Social. Un aspecto que ayudaría a este tipo de procesos de innovación y su interiorización por estudiantes y profesores fueron algunos de los "créditos cero" que pusieron en marcha algunas de las experiencias. Un lugar ideal para preparar al alumnado y compartir perspectivas de trabajo. También serviría para compartir estrategias de trabajo y búsqueda de información, formación procedimental y profesional, etc. Todo esto tiene sentido si existe un proceso de colaboración imprescindible entre el profesorado, estableciendo coherentemente la coordinación necesaria.

Nuevas formas de trabajo entre profesores

La forma de trabajar ayuda a imprimir el carácter necesario al cambio o la innovación, es fundamental que los profesores y profesoras empiecen a cambiar sus formas de trabajar centrados en procesos individuales y poco coordinados. Por tanto, la coordinación es imprescindible, siendo fundamental generar comisiones por titulación, en las que debe

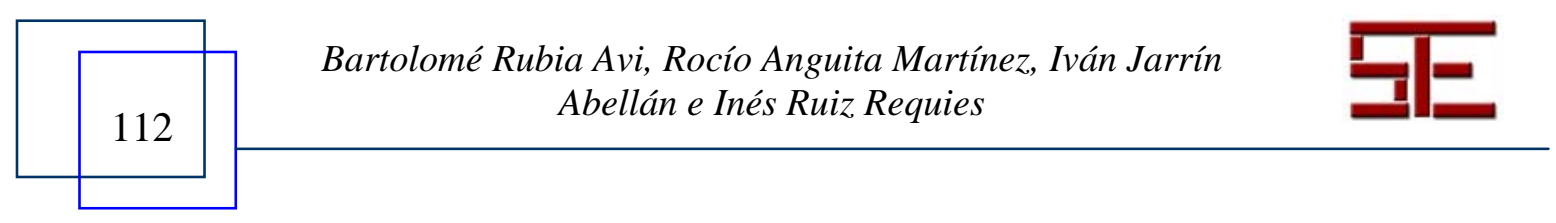




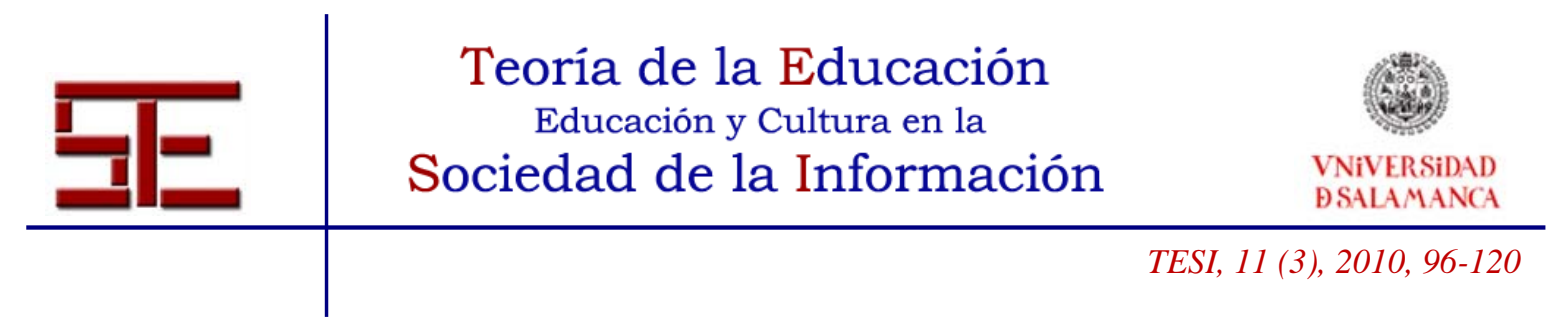

existir una coordinación de título y en las que esté presente el alumnado, así como la relación entre las distintas asignaturas. Esta forma de coordinación ha de surgir de nuevo de la creación de "comunidades de práctica” donde se puedan comunicar los miembros de la misma, respecto a los procesos y sus desarrollos. Donde se comparta lenguaje y aspiraciones, así como las ideas más rentables en la innovación dentro de una cultura de compromiso, necesaria para que lo individual forme parte de un proceso grupal coherente y eficaz.

\section{Modificación de tutorías}

Un elemento fundamental en las innovaciones, según se observa en las que hemos analizado y ayudado a generar, así como en la preocupación de las que conocemos fuera de nuestro entorno, tiene que ver con la manera nueva de contactar entre profesor y alumno. Las tutorías son el lugar donde el profesorado puede realizar una labor más eficaz de apoyo y seguimiento de las actividades formativas. Existe la necesidad de modificar las tutorías dándoles contenido, generando modalidades como las que surgieron de nuestras experiencias. Tutorías presenciales (grupales, individuales) en los espacios donde el profesor puede tener un contacto directo con el alumnado, pero no sólo en su despacho, sino en seminarios y tutorías grupales. Y también, tutorías virtuales apoyadas en un entorno tecnológico, que ayuden a resolver dudas y compartir reflexiones con el profesorado, de manera no presencial y en momentos de trabajo donde pueden coincidir ambos colectivos. Un concepto nuevo que surgió de nuestros grupos son las Tutorías temáticas, donde el profesor recoge aquellos aspectos donde confluyen dudas de los alumnos, organizando sesiones temáticas donde se analizan en años sucesivos. También entendimos que tendrían que ser una obligación para el alumnado, facilitando el necesario contacto con el profesor. Y, de manera fundamental, encontrar apoyo en las tecnologías para la generación de muchas de ellas.

\section{Cambio de materiales didácticos y documentos para el estudio}

Otro de los aspectos fundamentales de las innovaciones tiene que ver con la modificación de los materiales didácticos. Se impone el documento electrónico como alternativa a los clásicos en papel, entre otras cosas, por la cantidad que puede abarcar, así como la presencia perpetua de este tipo de recursos apoyados en plataformas y espacios virtuales.

También es fundamental la construcción de guías docentes donde quede estructurado el proceso formal de acción educativa, en el que contenidos, objetivos y competencias estén organizados para atender a la complejidad de la acción propuesta por la reforma educativa. Pero, a la vez, es necesario generar otra serie de guías que regulen los procesos no formales para facilitar la colaboración, los distintos procesos de trabajo, las ins-

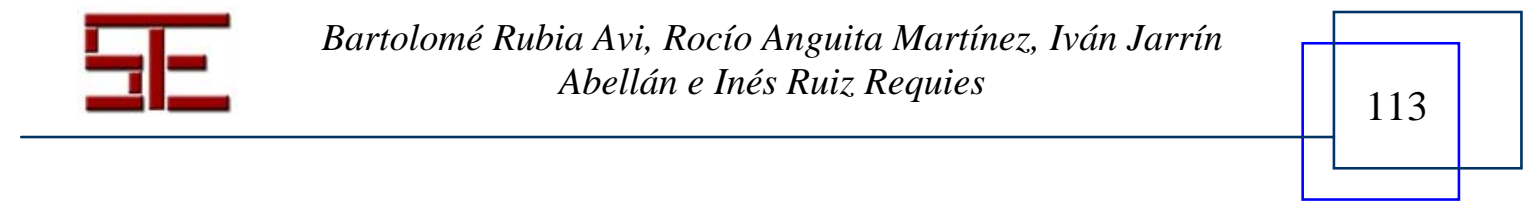




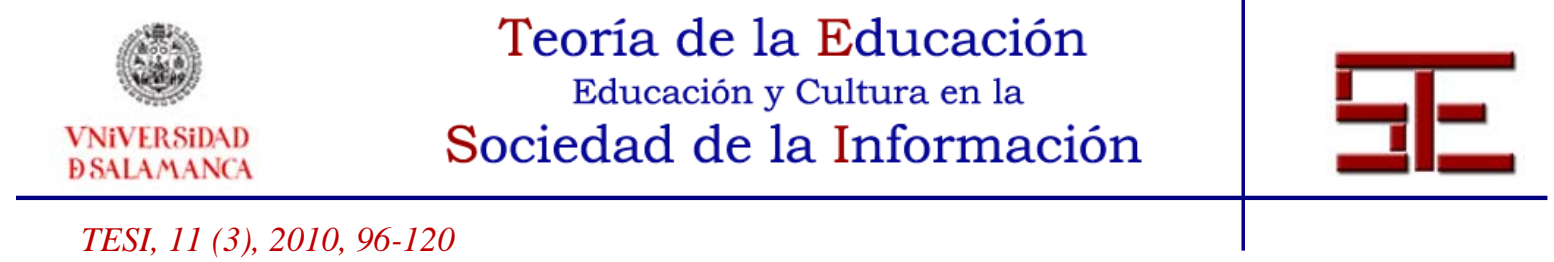

trucciones relacionadas con el fomento de tareas y procedimientos que ayuden a adquirir las destrezas transversales y a poner de acuerdo las instrucciones que debe compartir todo el profesorado.

Además de recursos para la docencia, es fundamental a veces recuperar y otras habilitar espacios en la Universidad para establecer lugares de encuentro e interacción. Tenemos que hacer de las facultades un lugar lleno de espacios para el trabajo, recuperando y habilitando pasillos, huecos de escalera, jardines... lugares donde sentarse a trabajar, conectar ordenadores con acceso Wifi. Lugares que ayuden a los tradicionales, aulas y bibliotecas, a facilitar la comunicación, el trabajo y la generación de tareas formativas grupales. Todo ello apoyado en formatos digitales para materiales docentes, así como para las tareas y trabajos del alumnado.

Nuevas formas de evaluación

Los procesos de innovación generan un incremento de la importancia de la evaluación, $\mathrm{y}$, por tanto, conllevan necesariamente una evaluación continua, puesto que continua es la actividad. Entre las modalidades de evaluación se entiende que:

- $\quad$ Es necesario pensar en formas de evaluar individualmente al alumnado dentro de su trabajo grupal.

- $\quad$ Evaluación por pares para facilitar la corresponsabilización en la evaluación y la asunción de la responsabilidad de ser evaluado.

- $\quad$ Evaluación por hitos en los que se deben constituir las distintas actividades formativas.

- $\quad$ Generar procesos de evaluación basados en trabajos dirigidos para el aprendizaje de conceptos y de procedimientos.

- $\quad$ Uso de procesos formales y puntuales de evaluación continuos y/o finales apoyados en los clásicos test.

- $\quad$ Actividades prácticas supervisadas en clase o de manera remota, así como de prácticas de laboratorio.

Es muy importante garantizar un feedback al alumnado tras sus aportaciones, para, de este modo, ver sus posibles errores e ir mejorando durante el proceso de formación.

\section{B. APORTACIONES A LA INTEGRACIÓN DE LAS TIC}

Este apartado relacionado con la innovación y las tecnologías, se apoya en el sentimiento que existe entre las personas que promueven cambios en la formación y que entienden que el apoyo de las TIC tiene que estar en el centro de los cambios.

Es fundamental disponer de recursos tecnológicos que faciliten el acceso a cualquier hora del día, los 7 días de la semana a los procesos de formación e innovación propues-

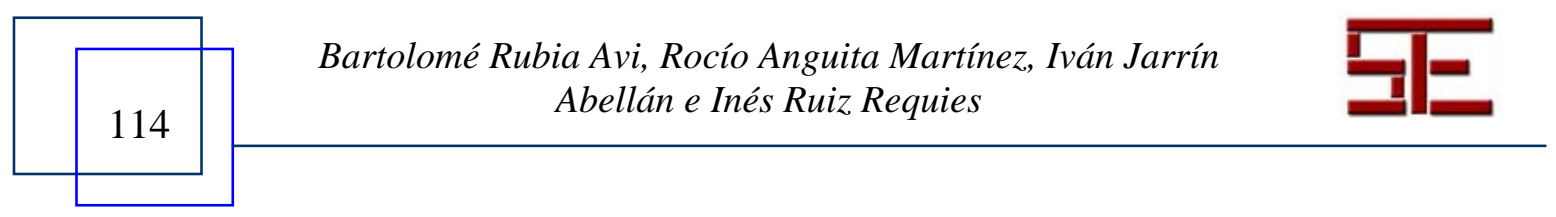




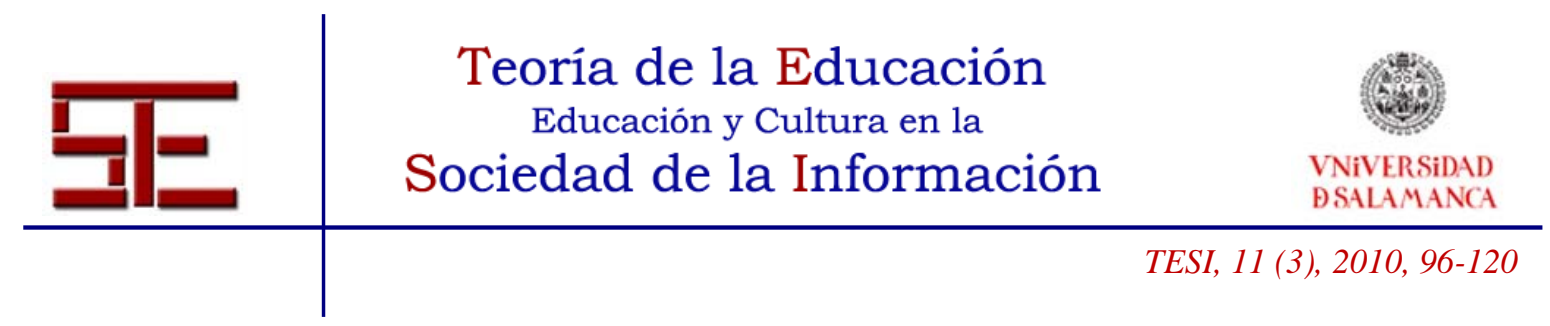

tos. Para esta cuestión, es fundamental la integración de todos los elementos curriculares de las programaciones de asignaturas dentro de espacios tecnológicos ágiles, sencillos y que abran posibilidades claras de interacción más allá de la mera exposición de contenidos. Necesitamos integrar tecnológicamente aspectos curriculares sobre las metodologías de trabajo grupal, donde el alumnado pueda compartir su trabajo, experiencias y reflexiones. Para esto, la tecnología ha de poder integrar: un debate activo sobre aspectos conceptuales de formación; un espacio compartido donde grupos de trabajo y colectivos más amplios puedan intercambiar procesos de elaboración; servicios de mensajerías y compartición de ficheros; espacios de relación con el profesorado; etc. Podemos decir, como aspecto fundamental, que la tecnología debe ponerse al servicio del proceso de enseñanza-aprendizaje, haciendo que sea fácil, rápido y on-line. Es fundamental crear centros de apoyo a la docencia como en los que se están convirtiendo algunos de los mencionados y relacionados con el cambio del EEES. Es fundamental la dotación económica que suponga un proceso de adquisición y de acceso a recursos, pero siempre a partir de la demanda de las personas implicadas en los cambios. Un centro de estas características tiene que superar la escasez de personal de apoyo, que no ha de centrarse solamente en técnicos informáticos que ayuden a mantener instalaciones, sino en la contratación de especialistas en formación y en tecnología que apoyen el diseño y desarrollo de entornos tecnológicos para la innovación. Este tipo de personal podría salir directamente de la propia comunidad universitaria, siguiendo el ejemplo de otros niveles educativos (educación infantil, primaria o secundaria) con los reconocidos y ya con mucha trayectoria, centros de profesores y recursos, que en Castilla y León se denomina CFIE (Centros de Formación e Innovación Educativa).

Y, como último aspecto, es fundamental la actualización continua, coordinada y racional de los recursos, debiendo facilitar la disponibilidad de accesos, ordenadores y servicios que faciliten todos estos procesos de formación y sus cambios, haciendo que la tecnología se integre en el proceso, como ahora están libros de texto, pizarras, papel y demás recursos clásicos.

Entendemos que todos estos aspectos plantean un elemento fundamental de adaptación de la Universidad a los cambios sociales, así como una preparación del alumnado para la vida después de la Universidad, donde las tecnologías forman parte importantísima de las destrezas y competencias para integrarse en ella. Por tanto, debemos transformar el uso lúdico de la tecnología, en uno formal.

\section{APORTACIONES AL MOVIMIENTO DE INNOVACIÓN EN LA UNIVERSIDAD}

A raíz de las distintas dinámicas de formación, experimentación y comunicación entre dichas experiencias, entendemos que procesos como éste proporcionan una nueva cultu-

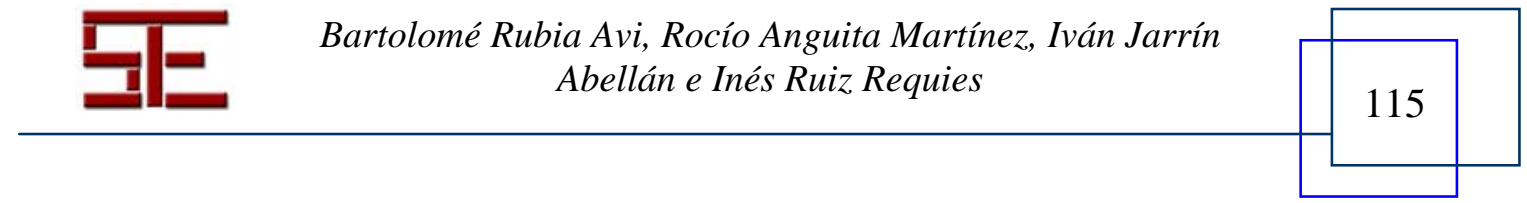




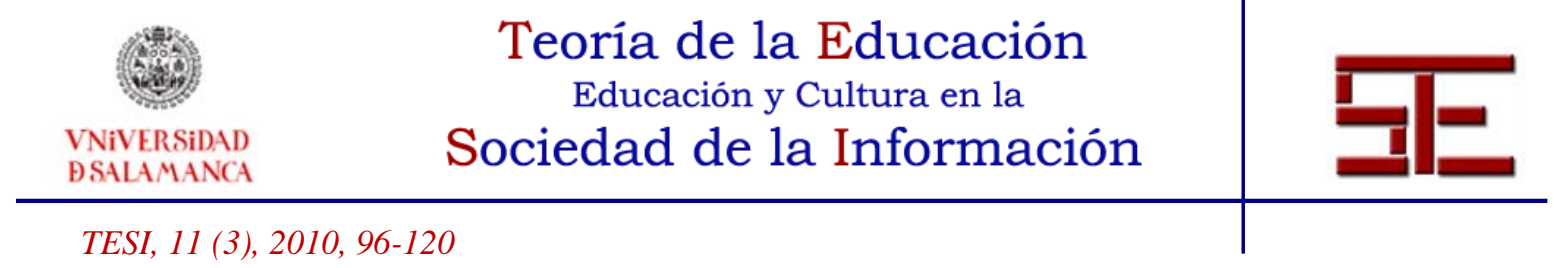

ra de la innovación para la mejora de la docencia y puesta en marcha del proceso de EEES. Estos procesos facilitan la generación de trayectorias y proyectos educativos que en distintas titulaciones han ido influyendo en esos cambios. Este proceso facilita que aumente el interés del profesorado por la generación de experiencias, de tal manera, que lo que surge del convencimiento tiene más trayectoria en un futuro. Es fundamental que las dinámicas de cambio se conviertan en innovaciones que impliquen a grupos más amplios, facilitando la generación de Proyectos Educativos en las distintas titulaciones de la Universidad. Entendidos como algo más profundo que la mera organización de contenidos y clases, sino como la conciencia sobre el tipo de profesionales que se está formando, definiendo un perfil claro y compartido por todo el centro donde se imparten. Dichos proyectos tienen que trasladar el espíritu de renovación, generando una acción unánime del profesorado apoyando el cambio. Esto marcará una trayectoria y un estilo docente compartido. Otro aspecto tiene que ver con las mejoras en las ratios profesorado/alumnado. Es fundamental que el profesorado siga de manera efectiva y eficiente la trayectoria de los alumnos, para eso, no podemos trabajar con grupos tan grandes.

Y como último aspecto significativo, entendemos que en los procesos de formación docente tiene que participar el PAS, puesto que las innovaciones no sólo ha de implementarlas el profesorado.

\section{6.- ALGUNAS REFLEXIONES SOBRE LA INNOVACIÓN Y LAS APORTA- CIONES DE LAS BUENAS PRÁCTICAS}

En este último apartado queremos reflejar la importancia de este trabajo que ha dado continuidad a múltiples esfuerzos realizados con anterioridad. Refleja un esfuerzo por definir una metodología colaborativa, que aunque no está reflejada en la descripción realizada en el artículo, sí que ha servido para presentar alguna de las ideas generales que ha ido desgranando el paso del tiempo. Son los grupos de personas, trabajando colaborativamente, los que producen estos cambios y mejoras en la formación y son estos grupos los que compartiendo entre ellos sus problemas, ideas y soluciones van configurando una manera compartida de hacer las cosas, que termina por generar el nuevo carácter de la Universidad y la formación que imparte.

También queremos resaltar como aportaciones, la importancia que tiene la historia acumulada y la relación entre iguales con una visión ética del trabajo, así como la toma de decisiones compartida. La tradición organizativa en la Universidad ha estado relacionada con una estructura verticalizada y donde la dinámica de formación estaba tomada por parte de los responsables de más alto nivel académico, entendemos que de manera mal entendida. Es fundamental que este tipo de cambios rompan con la organización vertical, la acción educativa en esta institución cumple los mismos requisitos prácticos que en el resto de los niveles educativos y, por tanto, o la democracia como organización y

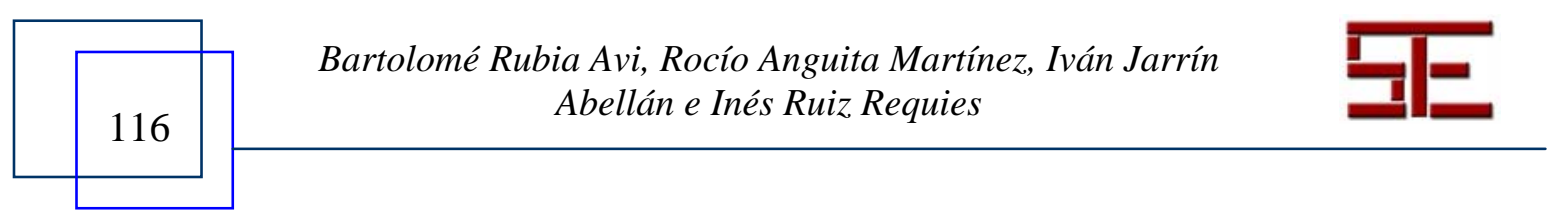




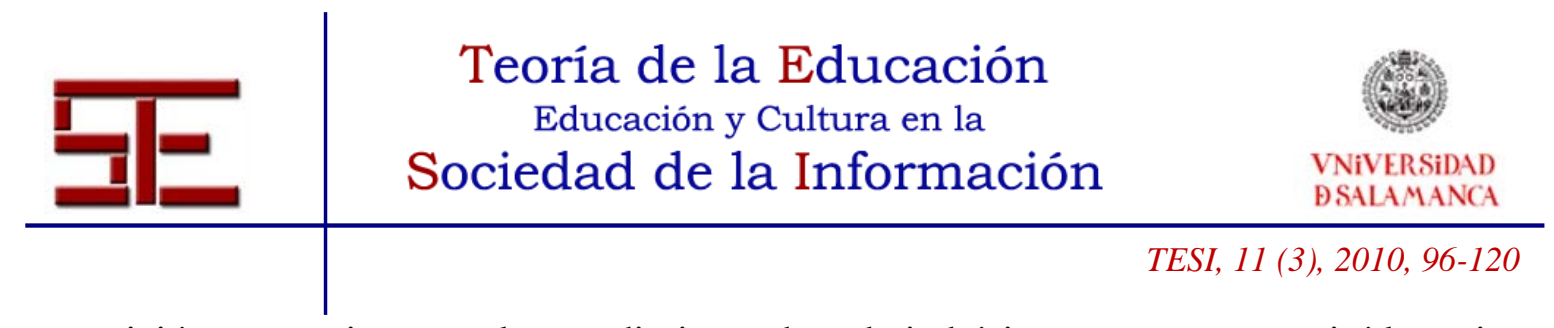

cognición se convierte en el procedimiento de trabajo básico o no se conseguirá la mejora y, sobre todo, la adaptación al funcionamiento de la sociedad.

Esto supone la introducción generalizada de la pedagogía como acción educativa, promovida por todas y cada una de las áreas de conocimiento, preocupación que debería tener la propia institución, además de estar definida como un elemento fundamental a la hora de promulgar planes de estudio, dinámicas organizativas, participación del profesorado en la definición de la docencia, así como en la identificación de los elementos tecnológicos y la configuración que ha de tener para la formación.

Para que todo esto se consiga, las “comunidades de práctica educativa” son la solución para el funcionamiento interno de las unidades docentes, donde aunque haya que definir mecanismos de coordinación interna y externa, éstas estén compuestas por personas iguales con toma de decisiones definitivamente horizontal.

En dichas comunidades, la decisión sobre los recursos también ha de estar cercana a la práctica educativa, por tanto, no podemos optar por decisiones tecnológicas en exceso generales. Que las Universidades adopten un soporte para permitir el apoyo tecnológico a la docencia es bueno, pero los campos de uso de esa tecnología tienen que tener definidas diferentes opciones tecnológicas en cuanto a extensiones y servicios en cada una de las opciones que contengan las plataformas tecnológicas que se escojan. Esto tiene que surgir de una política de generación de recursos a partir de las necesidades y no al contrario, que habitualmente primero definimos el espacio de plataforma y luego se termina adaptando la docencia a ese entorno o modelo tecnológico. Esto dificulta la diversidad en los modelos educativos que puedan pervivir en la Universidad, relacionados la mayoría de ellos con el tipo de materias y áreas de conocimiento.

También es fundamental el apoyo por parte de las universidades, de los desarrollos materiales en TIC que sean necesarios, si no apostamos por la inversión en medios, no se podrán habilitar los espacios para el cambio. Si el alumnado no tiene un servicio tecnológico necesario, el que sea más razonable económicamente y sin sobredimensionar el esfuerzo, no se podrá realizar ningún tipo de cambios. En algunas Universidades ya hace muchos años que al alumnado se le facilita el acceso a un ordenador portátil, cuando realizan su matrícula en primer curso, hay gente que dice que ésa no es la opción, pero sea la que tenga que ser, tiene que ver con un esfuerzo económico claro.

Y por último, decir que esta experiencia centrada en el análisis de las innovaciones supone un viaje por el paisaje de la formación y las inquietudes de cambio que las y los profesionales universitarios tienen, así como de sus necesidades. Esta foto fija sólo es un documento de trabajo que tiene valor si seguimos incidiendo en esta tarea de analizar

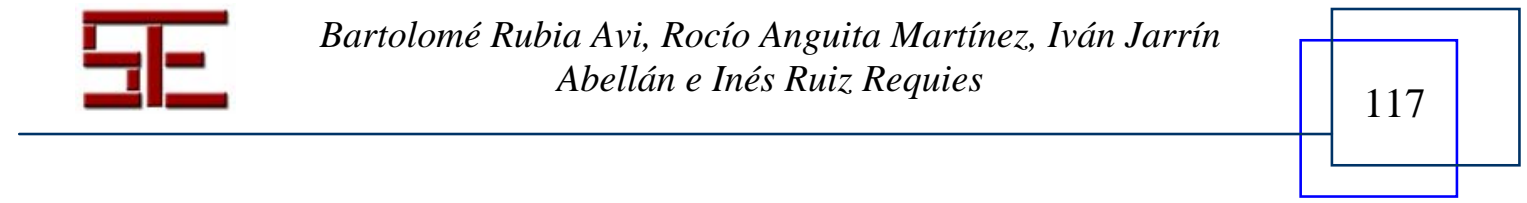




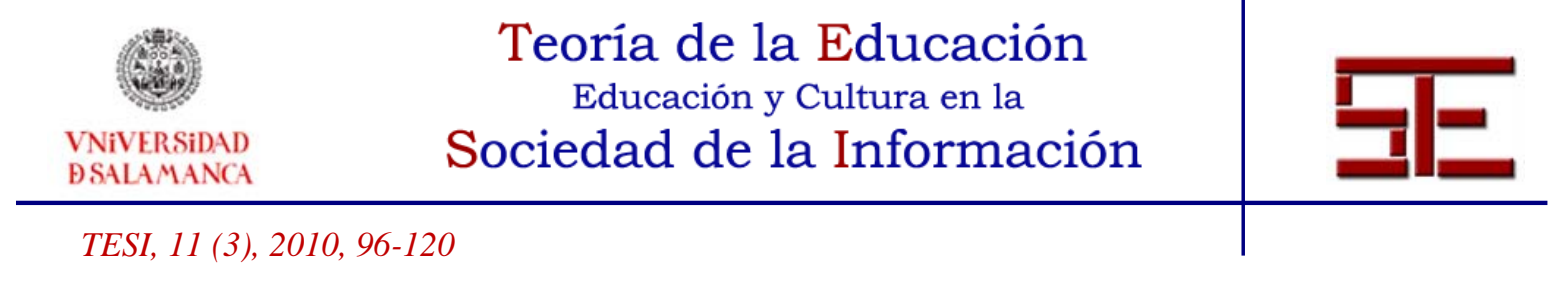

nuestra práctica educativa en la universidad, al igual que hacen multitud de docentes en otros niveles académicos.

\section{7.- BIBLIOGRAFÍA}

Bote-Lorenzo, M. L. et al. (2008). Gridcole: a tailorable grid service based system that supports scripted collaborative learning. Computers \& Education, 51(1), 155172.

Carr, W. (1996). Una teoría para la educación. Madrid: Ediciones Morata.

Connell, R. W. (2000). Escuelas, mercados, justicia: la educación en un mundo fracturado. Kikrirquí, 55/56, 4-13.

De la Calle, M. J. (2004). El reto de ser profesor en el contexto de la convergencia europea. La formación pedagógica como necesidad. RIFOP, 18 (3), 251-258.

Gómez-Sánchez, E. et al. (2002). Quest, a telematic tool for automatic management of student questionnaires in educational research. En Proc. of the Second European Conference on Technology, Information, Education and Citizenship. Barcelona, Spain.

González, M. A. et al. (2007). Evaluación de una experiencia de innovación docente para la adaptación de la Física I al EEES en una Escuela de Ingenieros Industriales. En Educatec 2007 (pp. 32-42). Buenos Aires: Educatec.

- (2008). Globalización y educación: ¿una amenazo o un reto? Laboratorios remotos, una herramienta deslocalizada para la mejora del aprendizaje. En Chafiqui Fouad, Pr., 15éme Congrès AMSE AMCE WAER sous patronage de l'UNESCO Mondialisation et éducaction vers une societé de la connaissance (Tomme II. Pp. 121-122). Marrakech: Recueil des résuimés des Sessions de Comunications Libres.

Hernández-Leo, D., et al. (2006). Collage, a Collaborative Learning Design Editor Based on Patterns Educational Technology \& Society. 9 (1), 58-71.

Jorrín Abellán, I. M., Rubia Avi, B. y Anguita Martínez, R. (2006). Formative Portrayals Emerged from a Computer Supported Collaborative Learning Environment: A Case Study to establish experiential generalizations. 2nd International congress of Qualitative Inquiry. Technology, Identity and Qualitative Inquiry session, Urbana-Champaign, Illinois, May, 112.

Jorrín Abellán, I. M. et al. (2006). A new formative pedagogical model emerged from the experience applicable to engineering courses based on CSCL. 36th Frontiers in Education Conference, T2C, 7-12. San Diego, CA, October.

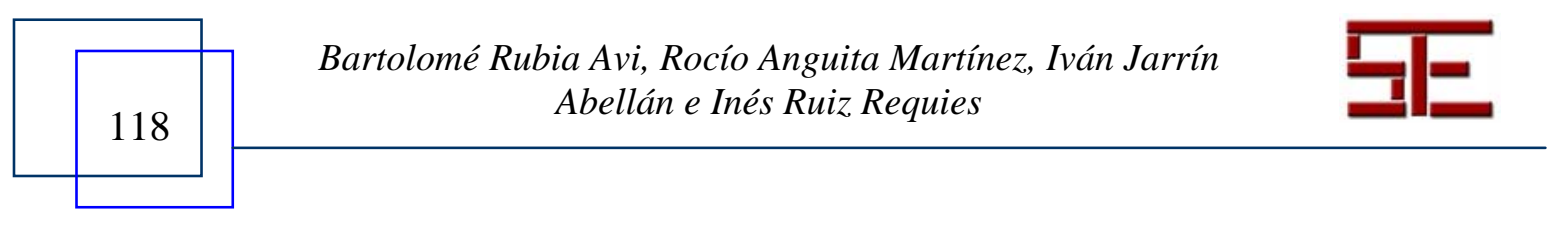




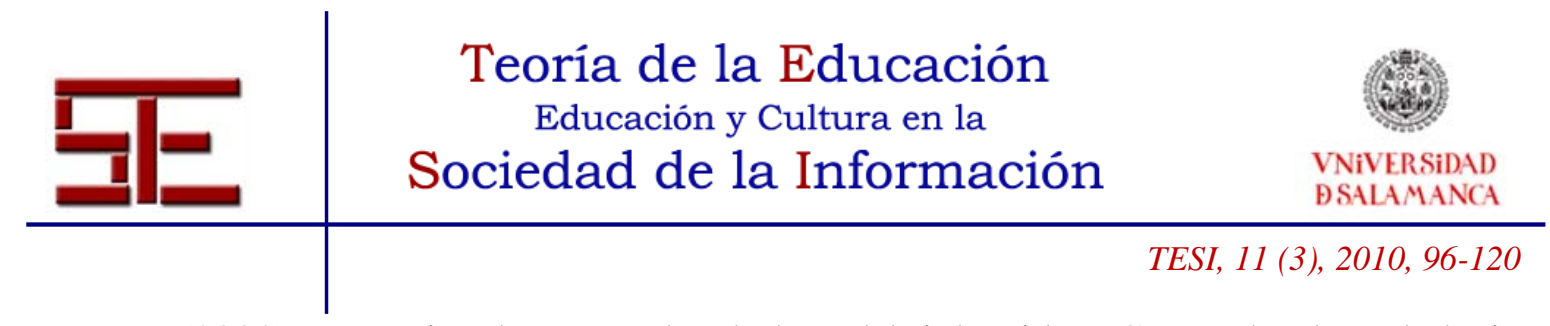

- (2008). Bouncing between the dark and bright sides: Can technology help in qualitative research? Journal of Qualitative Inquiry, Special Issue on Technology, 1187-1204.

Koschman, T. (1996). CSCL: Theory and Practice of an Emerging Paradigm. Mahwah, N.J.: Lawrence Erlbaum.

Martín, B. et al., (2007). Análisis y Evaluación de experiencias docentes innovadoras con e-learning llevadas a cabo en la asignatura de Física I en la E.T.S.I.I. En C. Guilarte Martín-Calero, (Eds.), Experiencias de innovación docente en la Universidad de Valladolid (pp. 387-400). Valladolid: Universidad de Valladolid.

Martínez, A. et al. (2003). Combining qualitative and social network analysis for the study of classroom social interactions. Computers and Education, 41(4), 353368.

Rubia Avi, B., Jorrín Abellán, I. M. (2004). Una experiencia de formación colaborativa y práctica real entre la Universidad y un centro educativo generando un espacio CSCL. Revista Latinoamericana de Tecnología Educativa, 1, 277-291.

Sáenz del Castillo, A. (1999). El (o) caso de los movimientos de renovación pedagógica (MRPs). Revista Electrónica Interuniversitaria de Formación del Profesorado, 2(1).

Salinas, J. (2004). Innovación docente y uso de las TIC en la enseñanza universitaria. Revista de Universidad y Sociedad del Conocimiento, 1, 1. [Disponible en: http://www.uoc.edu/rusc/dt/esp/salinas1104.pdf].

Sicilia, M. A., García-Barriocanal, E. y González-Sotos, L. (2006). Introducing fuzziness in object models and database interfaces through aspects. International Journal of Intelligent Systems. 21(12), 1199-1216.

Stenhouse, L. (1998). Investigacion y Desarrollo del Currículo. Madrid: Morata.

Toffler, A. (1985). The Adaptive Corporation. New York: McGraw Hill.

Vega-Gorgojo, G. et al. (2008). Ontoolcole: Supporting Educators in the Semantic Search of CSCL Tools. Journal of Universal Computer Science (JUCS), 14(1), 27-58.

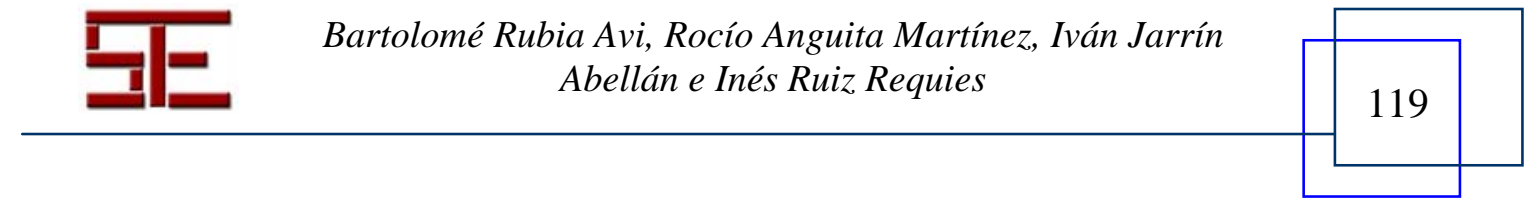


Para citar el presente artículo puede utilizar la siguiente referencia:

Rubia Avi, B., Anguita Martínez, R., Jarrín Abellán, I. y Ruiz Requies, I.: (2010). “Los procesos de innovación educativa en la formación universitaria, nuevos generadores de buenas prácticas en tecnología educativa”. En De Pablos Pons, J. (Coord.) Buenas prácticas de enseñanza con TIC [monográfico en línea]. Revista Electrónica Teoría de la Educación: Educación y Cultura en la Sociedad de la Información. Vol. 11, n ${ }^{0} 1$. Universidad de Salamanca, pp. 96-120. [Fecha de consulta: dd/mm/aaaa]. http://revistatesi.usal.es/ revistas_trabajo/index.php/revistatesi/article/view/5790/5820 ISSN: 1138-9737 